\title{
The Spreading Speed and the Existence of Planar Waves for a Class of Predator-prey System with Nonlocal Diffusion
}

\author{
Min Zhao*, Zhaohai Ma and Rong Yuan
}

\begin{abstract}
In this paper, we study a predator-prey system with general response function and nonlocal diffusion in high dimensional space and investigate the propagation properties of its solution. More precisely, we study the invasion speed of the predator into habitat of the aborigine prey and obtain the existence of the planar waves by constructing the upper and lower solutions. Finally, we present some numerical simulations to support our results.
\end{abstract}

\section{Introduction}

In recent years, some nonlocal diffusion population dynamics models have been established in order to interpret spatial variation of interactions between different populations, see [5, 11]. The study of dynamics of predator-prey interactions plays an important role in the theoretical ecology [8. Ducrot et al. 6] discussed the asymptotic speed of propagation and minimum wave speed of a predator-prey model with Holling type I response function in one-dimensional space. In this paper, we consider a class of predator-prey model with general response functions, which is more corresponding with practical biological significance. The predator-prey model with general functional response and nonlocal dispersal in high dimensional space can be expressed as follows:

$$
\left\{\begin{array}{rl}
\frac{\partial U}{\partial t}(x, t)= & d_{1} \mathcal{N}_{1}[U(\cdot, t)](x)+r_{1} U(x, t)(1-U(x, t)) \\
& -f(U(x, t)) V(x, t), \\
\frac{\partial V}{\partial t}(x, t)= & d_{2} \mathcal{N}_{2}[V(\cdot, t)](x)+\beta f(U(x, t)) V(x, t) \\
& -r_{2} V(x, t)(1+V(x, t)),
\end{array} \quad x \in \mathbb{R}^{N}, t>0 .\right.
$$

The system (1.1) is supplemented by the initial conditions:

$$
U(x, 0)=1, \quad V(x, 0)=v_{0}(x), \quad x \in \mathbb{R}^{N},
$$

Received April 14, 2021; Accepted September 30, 2021.

Communicated by Je-Chiang Tsai.

2020 Mathematics Subject Classification. 35K57, 35C07, 92D25.

Key words and phrases. predator-prey system, spreading speed, nonlocal diffusion, general response function.

*Corresponding author. 
where $v_{0}$ is a nonnegative continuous function with nonempty compact support. In system (1.1), $U=U(x, t)$ and $V=V(x, t)$ are the population densities of prey and predator species at time $t>0$ and the spatial position $x \in \mathbb{R}^{N}$, respectively. Here the dynamics of the prey population follow a logistic growth with a normalized (to one) carrying capacity and $r_{1}$ denotes its intrinsic growth rate. $f(U)$ is the functional response to the predator population. The constant $\beta$ is a conversion rate, $r_{2}$ is the death rate of predator species, $r_{1}>0, r_{2}>0$, and $\beta>0$. The term $d_{i} \mathcal{N}_{i}(i=1,2)$ describes the spatial dispersal of the individual. Here, $d_{1}>0$ and $d_{2}>0$ are the diffusion coefficients for prey and predator species, respectively. And $\mathcal{N}_{i}(i=1,2)$ is the linear nonlocal diffusion operators defined by

$$
\mathcal{N}_{i}[\varphi](x):=\left(J_{i} * \varphi\right)(x)-\varphi(x)=\int_{\mathbb{R}^{N}} J_{i}(x-y) \varphi(y) \mathrm{d} y-\varphi(x),
$$

where the symbol $*$ denotes the convolution product with respect to the spatial variable, and $J_{1}, J_{2}$ are probability kernel functions satisfying the following assumptions:

Assumption 1.1. The kernel function $J_{i}: \mathbb{R}^{N} \rightarrow \mathbb{R}(i=1,2)$ is a nonnegative radial continuous function and satisfies $\int_{\mathbb{R}^{N}} J_{i}(x) \mathrm{d} x=1, J_{i}(x)=J_{i}(-x)$ for all $x \in \mathbb{R}^{N}$ and there exists $\lambda_{0} \in(0,+\infty]$ such that $\int_{\mathbb{R}^{N}} J_{i}(x) \mathrm{e}^{\lambda x_{1}} \mathrm{~d} x<\infty$ for any $\lambda \in\left[0, \lambda_{0}\right)$.

To address more specifically the spreading speed and planar wave solutions of the system (1.1) with initial value conditions (1.2), we shall assume throughout this paper that the general functional response function satisfies certain assumptions, as follows:

Assumption 1.2. The function $f:[0, \infty) \rightarrow[0, \infty)$ is of class $C^{1}$ and satisfies:

(H1) $f(0)=0$ and $f(x)>0$ for any $x>0$;

(H2) $f^{\prime}(x)>0$ for any $x \in[0,+\infty)$;

(H3) $\frac{f(x)}{x}$ is continuous and monotonely nonincreasing for $x>0$.

Throughout this work, we shall assume the following parameter conditions:

$$
\beta f(1)>r_{2}, \quad a\left(\beta f(1)-r_{2}\right)<r_{1} r_{2},
$$

where $a$ is a positive constant satisfying $a \geq f^{\prime}(0)$.

It is well known that local diffusion models are often used to describe the spread of infectious diseases in space and the invasion of populations in biology. However, this local diffusion model can only be used to describe the diffusion phenomenon near a small area. In the actual environment, the movements of individuals are usually random and free 3,14 . Therefore, the nonlocal diffusion model is more realistic with the biological 
environment. In the theory of reaction-diffusion equations, asymptotic speed of propagation and planar wave solutions play an important role in describing biological invasion and disease transmission.

Many scholars have conducted a lot of research on spreading speed and traveling wave solutions in one-dimensional space, see $3,7,12,14,17,18$. In this paper, inspired by the processing of kernel functions in $[1,4,15]$, we mainly consider the spreading speed and planar wave solutions of predator-prey model with nonlocal diffusion and general response function in high-dimensional space. First, we consider the spreading speed of the predatory into habitat of the aborigine prey for model (1.1) with initial datum $(1.2)$. Then, we consider the existence of the planar wave solutions which connects the predator-free state $(1,0)$ with nontrivial state of the system (1.1). We will obtain that, under certain conditions, there exists $c^{*}>0$ such that for $c \geq c^{*}$, system (1.1) admits invasion planar wave solutions with wave speed $c$; while for $0<c<c^{*}$, system (1.1) has no invasion planar waves with wave speed $c$. We show that the spreading speed of the predatory is the minimal wave speed $c^{*}$ of the invasion planar waves.

This paper is organized as follows. In the next section, we establish some preliminary results. In Section 3, we give the proof of the spreading speed by using the comparison principle. In Section 4, we firstly present the proof of the existence of the planar wave solutions by constructing appropriate upper and lower solutions under the assumption of the compactly supported for the kernel function $J_{2}$. Then, we obtain the nonexistence of the invasion planar wave solutions. Finally, we have the result that the spreading speed of the predatory is the same as the minimal wave speed $c^{*}$. In Section 5 , we present some numerical simulations to support our results.

\section{Preliminaries}

Let

$$
X=\left\{w(x) \mid w(x): \mathbb{R}^{N} \rightarrow \mathbb{R} \text { is bounded and uniformly continuous }\right\}
$$

with norm

$$
\|w\|_{X}=\sup _{x \in \mathbb{R}^{N}}|w(x)|
$$

thus $\left(X,\|\cdot\|_{X}\right)$ is a Banach space. The positive cone $X^{+}$is defined by

$$
X^{+}=\left\{w \in X: w(x) \geq 0, \forall x \in \mathbb{R}^{N}\right\}
$$

Furthermore, for any constant $d>0$, let

$$
X_{d}=\left\{w \in X: 0 \leq w(x) \leq d, \forall x \in \mathbb{R}^{N}\right\}
$$


Set the order of the space $X^{2}=X \times X$ as follows:

$$
\underline{w} \leq \bar{w} \quad \Longleftrightarrow \quad \underline{w}_{i}(x) \leq \bar{w}_{i}(x), \quad x \in \mathbb{R}^{N}, i=1,2
$$

for any $\underline{w}=\left(\underline{w}_{1}(x), \underline{w}_{2}(x)\right)$ and $\bar{w}=\left(\bar{w}_{1}(x), \bar{w}_{2}(x)\right) \in X^{2}$.

We define the set $X_{H}^{2} \subset X^{2}$ by

$$
X_{H}^{2}=\left\{\left(w_{1}, w_{2}\right) \in X^{2}: 0 \leq w_{1} \leq 1 \text { and } 0 \leq w_{2} \leq \alpha\right\},
$$

where $\alpha:=\frac{\beta}{r_{2}} f(1)-1$. Our initial datum will always be chosen in this set. Here, we point out that under Assumption 1.2, the set $X_{H}^{2}$ is positively invariant under (1.1), therefore this system generates a strongly continuous nonlinear semiflow, denoted by $\mathbb{S}$, i.e., $\mathbb{S}=\left\{S(t): X_{H}^{2} \rightarrow X_{H}^{2}\right\}_{t \geq 0}$. In particular, this means that problem (1.1) with initial conditions 1.2 admits a unique globally defined solution $(U, V)$ with

$$
(U, V) \in C^{1}\left([0, \infty), X^{2}\right) \quad \text { and } \quad(U, V)(\cdot, t) \in X_{H}^{2}, \quad \forall t \geq 0 .
$$

Since $(U, V)$ is bounded from $[0, \infty)$ into $X^{2}$, it follows from 1.1 that the time derivative of $(U, V)$ is also bounded from $[0, \infty)$ into $X^{2}$. Hence, for each given initial data $v_{0} \in X_{\alpha}$, the corresponding solution $(U, V)=(U, V)(x, t)$ of system 1.1 with 1.2 is uniformly continuous on $\mathbb{R}^{N} \times[0, \infty)$. Furthermore, if $v_{0}$ admits a nonempty compact support, then $V(t, x)>0$ for all $t>0$ and $x \in \mathbb{R}^{N}$.

For our purpose, we would firstly like to present a comparison principle for the following equation with constant coefficients:

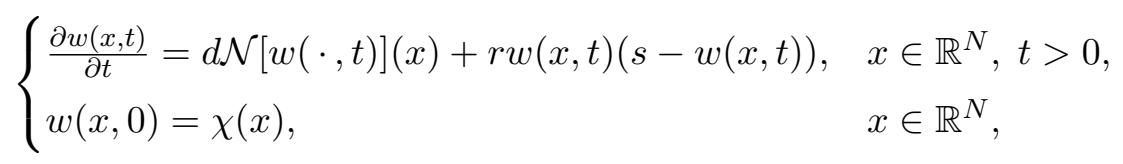

where $\mathcal{N}[w]:=J * w-w$, kernel $J$ satisfies Assumption 1.1 and the initial data $\chi \in X_{s}$ admits a nonempty compact support. The parameters satisfy $d>0, r>0$ and $s>0$. When $N=1$, Jin and Zhao [10] studied a scalar periodic logistic equation with nonlocal diffusion and established a comparison principle. To our knowledge, a comparison principle for general nonlocal diffusion equation in high dimensional space can not be found easily. For reader's convenience, we provide a proof for such equation.

Proposition 2.1. Let $w$ be a solution of (2.1) with $w(\cdot, t) \in X_{s}$ for all $t>0$ for a given $\chi \in X_{s}$. If $z(\cdot, t) \in X_{s}$ and $z(x, t)$ satisfies

$$
\begin{cases}\frac{\partial z(x, t)}{\partial t} \geq d \mathcal{N}[z(\cdot, t)](x)+r z(x, t)(s-z(x, t)), & x \in \mathbb{R}^{N}, t>0, \\ z(x, 0) \geq \chi(x), & x \in \mathbb{R}^{N},\end{cases}
$$

then $z(x, t) \geq w(x, t)$ for all $x \in \mathbb{R}^{N}, t>0$. Similar result holds for the reverse inequality. 
Proof. We prove the proposition by modifying some arguments of [10, Theorem 2.3]. Define $\kappa(x, t)=z(x, t)-w(x, t)$ for all $x \in \mathbb{R}^{N}, t>0$, and $\underline{\kappa}(t)=\inf _{x \in \mathbb{R}^{N}} \kappa(x, t)$ for all $t \geq 0$. Clearly, $\underline{\kappa}(t)$ is continuous in $t$ and $\underline{\kappa}(0) \geq 0$. We prove that $\underline{\kappa}(t) \geq 0$ for all $t \geq 0$. Indeed, by contradiction, we assume that for $\delta>0$, there exists $t_{0}>0$ such that $\underline{\kappa}\left(t_{0}\right)<0$ and

$$
\underline{\kappa}\left(t_{0}\right) e^{-\delta t_{0}}=\min _{t \in\left[0, t_{0}\right]} \underline{\kappa}(t) e^{-\delta t}<\underline{\kappa}(\tau) e^{-\delta \tau}, \quad \forall \tau \in\left[0, t_{0}\right) .
$$

It follows that there exists a sequence of points $\left\{x_{k}\right\}_{k=1}^{\infty}$ such that $\kappa\left(x_{k}, t_{0}\right)<0$ for all $k \geq 1$ and $\lim _{k \rightarrow \infty} \kappa\left(x_{k}, t_{0}\right)=\underline{\kappa}\left(t_{0}\right)$. Let $\left\{t_{k}\right\}_{k=1}^{\infty} \subseteq\left[0, t_{0}\right]$ be a sequence such that

$$
\kappa\left(x_{k}, t_{k}\right) e^{-\delta t_{k}}=\min _{t \in\left[0, t_{0}\right]} \kappa\left(x_{k}, t\right) e^{-\delta t} .
$$

Let $m_{\varepsilon}=\min _{t \in\left[0, t_{0}-\varepsilon\right]} \underline{\kappa}(t)^{e^{-\delta t}}$ for any $\varepsilon \in\left(0, t_{0}\right)$. Using 2.2), we obtain

$$
\lim _{k \rightarrow \infty} \kappa\left(x_{k}, t_{0}\right) e^{-\delta t_{0}}=\underline{\kappa}\left(t_{0}\right) e^{-\delta t_{0}}<m_{\varepsilon} .
$$

Thus, there exists an integer $K_{\varepsilon}$ such that for all $k \geq K_{\varepsilon}$,

$$
\kappa\left(x_{k}, t_{0}\right) e^{-\delta t_{0}}<m_{\varepsilon} \leq \underline{\kappa}(t) e^{-\delta t} \leq \kappa\left(x_{k}, t\right) e^{-\delta t}, \quad \forall t \in\left[0, t_{0}-\varepsilon\right] .
$$

By 2.3), we have

$$
\kappa\left(x_{k}, t_{k}\right) e^{-\delta t_{k}}=\min _{t \in\left[0, t_{0}\right]} \kappa\left(x_{k}, t\right)^{e^{-\delta t}} \leq \kappa\left(x_{k}, t_{0}\right) e^{-\delta t_{0}},
$$

thus, $t_{k} \in\left[t_{0}-\varepsilon, t_{0}\right]$ for all $k \geq K_{\varepsilon}$. Then we deduce that $\lim _{k \rightarrow \infty} t_{k}=t_{0}$. Due to

$$
\kappa\left(x_{k}, t_{0}\right) e^{-\delta t_{0}} \geq \kappa\left(x_{k}, t_{k}\right) e^{-\delta t_{k}} \geq \underline{\kappa}\left(t_{k}\right) e^{-\delta t_{k}} \geq \underline{\kappa}\left(t_{0}\right) e^{-\delta t_{0}},
$$

we get

$$
\kappa\left(x_{k}, t_{0}\right) e^{-\delta\left(t_{0}-t_{k}\right)} \geq \kappa\left(x_{k}, t_{k}\right) \geq \underline{\kappa}\left(t_{0}\right) e^{-\delta\left(t_{0}-t_{k}\right)} .
$$

Letting $k \rightarrow \infty$, we have $\lim _{k \rightarrow \infty} \kappa\left(x_{k}, t_{k}\right)=\underline{\kappa}\left(t_{0}\right)$. Then 2.3$)$ implies that

$$
0 \geq\left.\frac{\partial\left(\kappa\left(x_{k}, t\right) e^{-\delta t}\right)}{\partial t}\right|_{t=t_{k}^{-}}=e^{-\delta t_{k}}\left(\frac{\partial \kappa\left(x_{k}, t_{k}\right)}{\partial t}-\delta \kappa\left(x_{k}, t_{k}\right)\right) .
$$

Thus

$$
\frac{\partial \kappa\left(x_{k}, t_{k}\right)}{\partial t} \leq \delta \kappa\left(x_{k}, t_{k}\right)
$$

The function $g: \mathbb{R} \rightarrow \mathbb{R}$ is denoted

$$
g(l):=r l(s-l),
$$

where $l:=l(x, t), \forall x \in \mathbb{R}^{N}, t \geq 0$. By the definition of $g$, for every $M_{0}>0$, there exists constant $L>0$ such that

$$
|g(z)-g(w)| \leq L|z-w|, \quad \forall z, w \in\left[0, M_{0}\right]
$$


Because $\kappa\left(x_{k}, t_{k}\right)<0$ and by $(2.4)$, it follows that

$$
\begin{aligned}
\frac{\partial \kappa\left(x_{k}, t_{k}\right)}{\partial t} & =\frac{\partial z\left(x_{k}, t_{k}\right)}{\partial t}-\frac{\partial w\left(x_{k}, t_{k}\right)}{\partial t} \\
& \geq d \mathcal{N}\left[\kappa\left(\cdot, t_{k}\right)\right]\left(x_{k}\right)+g\left(z\left(x_{k}, t_{k}\right)\right)-g\left(w\left(x_{k}, t_{k}\right)\right) \\
& \geq d\left[\int_{\mathbb{R}^{N}} J\left(x_{k}-y\right) \kappa\left(y, t_{k}\right) \mathrm{d} y-\kappa\left(x_{k}, t_{k}\right)\right]+L \kappa\left(x_{k}, t_{k}\right) .
\end{aligned}
$$

Then

$$
\begin{aligned}
0 & \leq \frac{\partial \kappa\left(x_{k}, t_{k}\right)}{\partial t}-L \kappa\left(x_{k}, t_{k}\right)-d\left[\int_{\mathbb{R}^{N}} J\left(x_{k}-y\right) \kappa\left(y, t_{k}\right) \mathrm{d} y-\kappa\left(x_{k}, t_{k}\right)\right] \\
& \leq(\delta-L+d) \kappa\left(x_{k}, t_{k}\right)-d \underline{\kappa}\left(t_{k}\right) .
\end{aligned}
$$

Letting $k \rightarrow \infty$, we obtain $0 \leq(\delta-L) \underline{\kappa}\left(t_{0}\right)$. For $\delta>L$, it follows that $\underline{\kappa}\left(t_{0}\right) \geq 0$, a contradiction. Therefore, we get $\underline{\kappa}(t) \geq 0$ for any $t \geq 0$. Thus, we obtain $\kappa(x, t) \geq 0$, i.e.,

$$
z(x, t) \geq w(x, t) \quad \text { for all } x \in \mathbb{R}^{N}, t \geq 0 .
$$

We complete the proof.

Remark 2.2. Through the proof of Proposition 2.1, we can generalize its conclusion to a more general system:

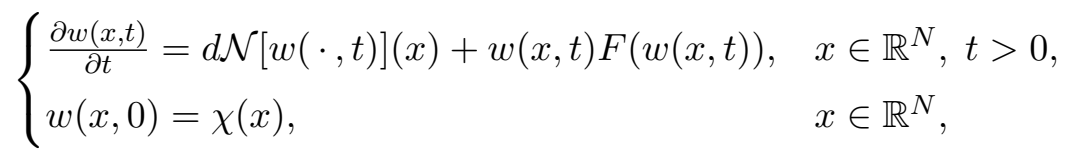

where $F \in C^{1}(\mathbb{R}, \mathbb{R}), F_{w}<0$ for all $w \in \mathbb{R}$, we have $z(x, t) \geq w(x, t)$ for all $x \in \mathbb{R}^{N}, t>0$, where $z(x, t)$ satisfies

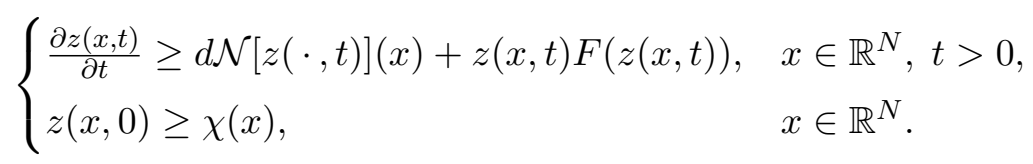

Moreover, Jin and Zhao [10] studied the spreading speed of a scalar periodic logistic equation with nonlocal diffusion in one-dimensional space. Diekmann 4 investigated the spreading speed of the nonlinear integral equation of mixed Volterra-Fredholm type in high-dimensional case. Ma 13 explored the asymptotic speed of spread of a general nonlocal diffusion equation in $n$-dimensional case. Based on their research, we conclude that system (2.1) has the following spreading speed.

Proposition 2.3. Let $w$ be a solution of (2.1) with $w(\cdot, t) \in X_{s}$ for all $t>0$ for a given $\chi \in X_{s}$ and $\bar{c}:=\inf _{0<\lambda<\lambda_{0}} \frac{d\left[\int_{\mathbb{R}^{N}} J(x) \mathrm{e}^{\lambda x_{1}} \mathrm{~d} x-1\right]+r s}{\lambda}>0$. Then the following statements are valid. 
(i) For any $c>\bar{c}$, if $\chi$ has a nonempty compact support, then

$$
\lim _{t \rightarrow \infty} \sup _{\|x\|>c t} w(x, t)=0
$$

(ii) For any $0<c<\bar{c}$, if $\chi(\cdot) \not \equiv 0$, then

$$
\liminf _{t \rightarrow \infty} \inf _{\|x\|<c t} w(x, t)=s .
$$

Since the kernel function $J_{2}$ satisfies Assumption 1.1, we define

$$
c^{*}:=\inf _{0<\lambda<\lambda_{0}} \frac{d_{2}\left[\int_{\mathbb{R}^{N}} J_{2}(x) \mathrm{e}^{\lambda x_{1}} \mathrm{~d} x-1\right]+\beta f(1)-r_{2}}{\lambda} .
$$

\section{Spreading speed}

In this section, we will discuss the spreading speed of the predator for system (1.1) with initial values $(1.2)$ and establish the following theorem.

Theorem 3.1. Under the assumption (1.3), the constant $c^{*}$, defined in (2.6), corresponds to the spreading speed of the predator for system (1.1) with initial datum (1.2) and $0 \leq$ $v_{0} \leq \alpha$. This means that the density of the predator $V=V(x, t)$ satisfies the following statements.

(1) For any $c>c^{*}$, if $v_{0}$ is a nonzero compactly supported continuous function, then

$$
\lim _{t \rightarrow \infty} \sup _{\|x\|>c t} V(x, t)=0
$$

(2) For any $0<c<c^{*}$, if $v_{0}(\cdot) \not \equiv 0$, then

$$
\liminf _{t \rightarrow \infty} \inf _{\|x\|<c t} V(x, t)>0 .
$$

Proof. First, we prove that

$$
\lim _{t \rightarrow \infty} \sup _{\|x\|>c t} V(x, t)=0 \text { for any } c>c^{*} .
$$

Since $U \leq 1$, using (H2), we get that $f(U) \leq f(1)$ and the function $V$ satisfies

$$
\frac{\partial V(x, t)}{\partial t} \leq d_{2} \mathcal{N}_{2}[V(\cdot, t)](x)+\beta f(1) V(x, t)-r_{2} V(x, t)(1+V(x, t)), \quad x \in \mathbb{R}^{N}, t>0 .
$$

By Proposition 2.1, for all $(x, t) \in \mathbb{R}^{N} \times[0, \infty)$, we have $V(x, t) \leq \bar{V}(x, t)$, where $\bar{V}$ satisfies

$$
\left\{\begin{array}{l}
\frac{\partial \bar{V}(x, t)}{\partial t}=d_{2} \mathcal{N}_{2}[\bar{V}(\cdot, t)](x)+\beta f(1) \bar{V}(x, t)-r_{2} \bar{V}(x, t)(1+\bar{V}(x, t)), \quad x \in \mathbb{R}^{N}, t>0, \\
\bar{V}(x, 0)=v_{0}(x) .
\end{array}\right.
$$


Hence, according to 2.5 in Proposition 2.3, we obtain (3.1).

Now we prove the statement (2), namely

$$
\liminf _{t \rightarrow \infty} \inf _{\|x\|<c t} V(x, t)>0 \quad \text { for any given } c \in\left(0, c^{*}\right) .
$$

According to (H2) and (H3), we deduce that $f(x) \leq a x$, where $a \geq f^{\prime}(0)>0$. Since $V(x, t) \leq \alpha$, the function $U$ satisfies

$$
\frac{\partial U(x, t)}{\partial t} \geq d_{1} \mathcal{N}_{1}[U(\cdot, t)](x)+U(x, t)\left(r_{1}-a \alpha-r_{1} U(x, t)\right), \quad x \in \mathbb{R}^{N}, t>0 .
$$

Hence, according to the second condition in 1.3$)$, i.e., $a\left(\beta f(1)-r_{2}\right)<r_{1} r_{2}$ and $U(x, 0)=1$, we derive from Proposition 2.1 that

$$
U(x, t) \geq U_{\min }:=1-\frac{a}{r_{1} r_{2}}\left(\beta f(1)-r_{2}\right), \quad \forall x \in \mathbb{R}^{N}, t \geq 0 .
$$

Also using (3.2), $U \leq 1$ and (H2), the function $U$ satisfies

$$
\frac{\partial U(x, t)}{\partial t} \geq d_{1} \mathcal{N}_{1}[U(\cdot, t)](x)+r_{1} U_{\min }(1-U(x, t))-f(1) V(x, t), \quad x \in \mathbb{R}^{N}, t>0 .
$$

Because $U(x, 0)=1$, the function $1-U(x, t)$ satisfies

$$
1-U(x, t) \leq \bar{u}(x, t):=f(1) \int_{0}^{t} \exp \left(-r_{1} U_{\min }(t-s)\right) \exp \left((t-s) d_{1} \mathcal{N}_{1}\right)[V(\cdot, s)](x) \mathrm{d} s
$$

for all $x \in \mathbb{R}^{N}, t \geq 0$. By (H3), we deduce that $f(U) \geq-f(1) \bar{u}+f(1)$. Plugging (3.3) into the $V$-equation in 1.1 yields

$$
\begin{aligned}
\frac{\partial V(x, t)}{\partial t} \geq & d_{2} \mathcal{N}_{2}[V(\cdot, t)](x) \\
& +V(x, t)\left[-\beta f(1) \bar{u}(x, t)-r_{2} V(x, t)-r_{2}+\beta f(1)\right], \quad x \in \mathbb{R}^{N}, t>0 .
\end{aligned}
$$

Let $\epsilon \in\left(0, c^{*}\right)$ be given, $\delta_{0}>0$ small enough and $\delta_{0}<\beta f(1)-r_{2}$ such that

$$
\inf _{0<\lambda<\lambda_{0}} \frac{d_{2}\left[\int_{\mathbb{R}^{N}} J_{2}(x) \mathrm{e}^{\lambda x_{1}} \mathrm{~d} x-1\right]+\beta f(1)-r_{2}-\delta_{0}}{\lambda}>c^{*}-\epsilon .
$$

Next, we claim there exist $M>0$ and $\tau>0$ large enough such that

$$
\beta f(1) \bar{u}(x, t) \leq \delta_{0}+M V(x, t), \quad x \in \mathbb{R}^{N}, t \geq \tau .
$$

Indeed, to prove the claim, it suffices to show that there exists some constant $M>0$ such that, for any $t \geq \tau$ and $x \in \mathbb{R}^{N}$, we have

$$
\beta f(1) \bar{u}(x, t) \geq \delta_{0} \quad \Longrightarrow \quad \beta f(1) \bar{u}(x, t) \leq \delta_{0}+M V(x, t) .
$$


To the aim, we consider the strongly positive semigroup $\{T(t)\}_{t \geq 0}$, where

$$
T(t):=\exp \left(t d_{1} \mathcal{N}_{1}\right), \quad t \geq 0 \text {. }
$$

According to [9], we know that the solution $W$ of the problem

$$
\frac{\partial w(x, t)}{\partial t}=d_{1}\left\{\left[J_{1} * w(\cdot, t)\right](x)-w(x, t)\right\}, \quad t>0 \text { with } w(\cdot, 0)=\delta_{1},
$$

where $\delta_{1}$ denotes the Dirac mass at $x=0$, can be decomposed as

$$
W(x, t)=\mathrm{e}^{-d_{1} t} \delta_{1}(x)+K(x, t), \quad x \in \mathbb{R}^{N}, t \geq 0,
$$

where $K$ is a nonnegative smooth function satisfying the estimate

$$
\int_{\mathbb{R}^{N}} K(x, t) \mathrm{d} x \leq 2, \quad \forall t \geq 0 .
$$

Thus, the semigroup $\{T(t)\}_{t \geq 0}$ can be expressed as

$$
T(t)[\varphi](x)=\mathrm{e}^{-d_{1} t} \varphi(x)+\int_{\mathbb{R}^{N}} K(x-y, t) \varphi(y) \mathrm{d} y, \quad \forall t \geq 0, \varphi \in X .
$$

Using the above formula for $T(t)$, the function $\beta f(1) \bar{u}(x, t)$ can be decomposed as $\beta f(1)$ $\bar{u}(x, t)=W_{1}(x, t)+W_{2}(x, t)$, where

$$
\begin{gathered}
W_{1}(x, t):=a \beta f(1) \int_{0}^{t} \mathrm{e}^{-\beta_{1}(t-s)} V(x, s) \mathrm{d} s, \quad \beta_{1}:=d_{1}+r_{1} U_{\min }>0, \\
W_{2}(x, t):=a \beta f(1) \int_{0}^{t} \int_{\mathbb{R}^{N}} \mathrm{e}^{-\beta_{2}(t-s)} K(x-y, t-s) V(y, s) \mathrm{d} y \mathrm{~d} s, \quad \beta_{2}:=r_{1} U_{\min }>0 .
\end{gathered}
$$

Since $\delta_{0}>0$ is fixed, there exists $\tau>0$ large enough such that

$$
a \beta f(1) \alpha \int_{0}^{t-\tau} \mathrm{e}^{-\beta_{1}(t-s)} \mathrm{d} s \leq \frac{\delta_{0}}{4}, \quad 2 a \beta f(1) \alpha \int_{0}^{t-\tau} \mathrm{e}^{-\beta_{2}(t-s)} \mathrm{d} s \leq \frac{\delta_{0}}{4}, \quad \forall t \geq \tau .
$$

In order to prove (3.6), we consider $t_{0} \geq \tau$ and $x_{0} \in \mathbb{R}^{N}$ with $\beta f(1) \bar{u}\left(x_{0}, t_{0}\right) \geq \delta_{0}$. First, since $V \leq \alpha$, from $(3.7)$, it follows that

$$
\begin{aligned}
\delta_{0} \leq \beta f(1) \bar{u}\left(x_{0}, t_{0}\right) \leq & a \beta f(1) \alpha \int_{0}^{t_{0}-\tau} \mathrm{e}^{-\beta_{1}\left(t_{0}-s\right)} \mathrm{d} s+2 a \beta f(1) \alpha \int_{0}^{t_{0}-\tau} \mathrm{e}^{-\beta_{2}\left(t_{0}-s\right)} \mathrm{d} s \\
& +a \beta f(1) \int_{t_{0}-\tau}^{t_{0}} \mathrm{e}^{-\beta_{1}\left(t_{0}-s\right)} V\left(x_{0}, s\right) \mathrm{d} s \\
& +a \beta f(1) \int_{t_{0}-\tau}^{t_{0}} \int_{\mathbb{R}^{N}} \mathrm{e}^{-\beta_{2}\left(t_{0}-s\right)} K\left(x_{0}-y, t_{0}-s\right) V(y, s) \mathrm{d} y \mathrm{~d} s
\end{aligned}
$$

This combined with 3.8 , yields

$$
a \beta f(1) \int_{0}^{\tau} \mathrm{e}^{-\beta_{1} l} V\left(x_{0}, t_{0}-l\right) \mathrm{d} l+a \beta f(1) \int_{0}^{\tau} \int_{\mathbb{R}^{N}} \mathrm{e}^{-\beta_{2} l} K(y, l) V\left(x_{0}-y, t_{0}-l\right) \mathrm{d} y \mathrm{~d} l \geq \frac{\delta_{0}}{2} .
$$


Next, choose $r>0$ large enough such that

$$
a \beta f(1) \alpha \int_{0}^{\tau} \int_{\|y\| \geq r} \mathrm{e}^{-\beta_{2} l} K(y, l) \mathrm{d} y \mathrm{~d} l \leq \frac{\delta_{0}}{4} .
$$

According to $V \leq \alpha$, it follows that

$$
a \beta f(1) \int_{0}^{\tau} \mathrm{e}^{-\beta_{1} l} V\left(x_{0}, t_{0}-l\right) \mathrm{d} l+a \beta f(1) \int_{0}^{\tau} \int_{\|y\| \leq r} \mathrm{e}^{-\beta_{2} l} K(y, l) V\left(x_{0}-y, t_{0}-l\right) \mathrm{d} y \mathrm{~d} l \geq \frac{\delta_{0}}{4} .
$$

Let $\eta>0$ small enough such that

$$
a \beta f(1) \alpha\left[\int_{0}^{\eta} \mathrm{e}^{-\beta_{1} l} \mathrm{~d} l+\int_{0}^{\eta} \int_{\|y\| \leq r} \mathrm{e}^{-\beta_{2} l} K(y, l) \mathrm{d} y \mathrm{~d} l\right] \leq \frac{\delta_{0}}{8} .
$$

Thus, using the same argument as above, it follows that

$$
a \beta f(1) \int_{\eta}^{\tau} \mathrm{e}^{-\beta_{1} l} V\left(x_{0}, t_{0}-l\right) \mathrm{d} l+a \beta f(1) \int_{\eta}^{\tau} \int_{\|y\| \leq r} \mathrm{e}^{-\beta_{2} l} K(y, l) V\left(x_{0}-y, t_{0}-l\right) \mathrm{d} y \mathrm{~d} l \geq \frac{\delta_{0}}{8} .
$$

Setting $\theta>0$ by

$$
\frac{\delta_{0}}{8}=\theta\left[a \beta f(1) \int_{\eta}^{\tau} \mathrm{e}^{-\beta_{1} l} \mathrm{~d} l+a \beta f(1) \int_{\eta}^{\tau} \int_{\|y\| \leq r} \mathrm{e}^{-\beta_{2} l} K(y, l) \mathrm{d} y \mathrm{~d} l\right],
$$

it follows that there are $l_{0} \in\left[t_{0}-\tau, t_{0}-\eta\right]$ and $y_{0} \in B_{r}\left(x_{0}\right)$ such that $V\left(y_{0}, l_{0}\right) \geq \theta$. Furthermore, since the function $V$ is uniformly continuous on $\mathbb{R}^{N} \times[0, \infty)$, there exists $\rho>0$ independent of $\left(y_{0}, l_{0}\right)$ such that

$$
V\left(y, l_{0}\right) \geq \frac{\theta}{2}, \quad \forall y \in \bar{B}_{\rho}\left(y_{0}\right),
$$

where $\bar{B}$ represents the closure of $B$. Finally, we define a uniformly continuous function $Z_{0} \leq \frac{\theta}{2}$ in $\mathbb{R}$ such that

$$
Z_{0}(x)=\frac{\theta}{2}, \quad \forall x \in \bar{B}_{\frac{\rho}{2}}(0), \quad Z_{0}(x)=0, \quad \forall x \in \mathbb{R}^{N} \backslash \bar{B}_{\rho}(0) .
$$

Clearly, the function $V$ satisfies

$$
\frac{\partial V(x, t)}{\partial t} \geq d_{2} \mathcal{N}_{2}[V(\cdot, t)](x)-r_{2}(1+\alpha) V(x, t), \quad x \in \mathbb{R}^{N}, t>0 .
$$

Since $Z_{0}(x) \leq V\left(y_{0}+x, l_{0}\right)$, according to the comparison principle, we obtain

$$
Z(x, t) \leq V\left(y_{0}+x, l_{0}+t\right), \quad \forall x \in \mathbb{R}^{N}, t \geq 0,
$$

where $Z(\cdot, t)=\mathrm{e}^{r_{2}(1+\alpha) t} \exp \left(t d_{2} \mathcal{N}_{2}\right)\left[Z_{0}\right](\cdot)$. Clearly $Z(x, t)>0$ for all $x \in \mathbb{R}^{N}, t>0$. Thus, we get that

$$
V\left(x_{0}, t_{0}\right) \geq Z\left(x_{0}-y_{0}, t_{0}-l_{0}\right) \geq \gamma:=\min _{x \in \bar{B}_{r}(0), t \in[\eta, \tau]} Z(x, t)>0 .
$$


Then, using (3.7), 3.8), 3.9) and $V \leq \alpha$, we have that

$$
\beta f(1) \bar{u}\left(x_{0}, t_{0}\right)<\frac{\delta_{0}}{2}+3 a \beta f(1) \alpha \tau \leq \delta_{0}+M V\left(x_{0}, t_{0}\right),
$$

where we set $M=3 \gamma^{-1} a \beta f(1) \alpha \tau>0$. Clearly, $M$ is independent of $\left(x_{0}, t_{0}\right)$. This completes the proof of the claim.

Inserting (3.5) into (3.4) yields

$\frac{\partial V(x, t)}{\partial t} \geq d_{2} \mathcal{N}_{2}[V(\cdot, t)](x)+V(x, t)\left[\beta f(1)-r_{2}-\delta_{0}-\left(r_{2}+M\right) V(x, t)\right], \quad x \in \mathbb{R}^{N}, t>\tau$.

According to the comparison principle, it follows that $V(x, t+\tau) \geq \underline{V}(x, t)$ for any $x \in \mathbb{R}^{N}$, $t \geq 0$, where the function $\underline{V}$ is a solution of the scalar logistic equation

$$
\left\{\begin{aligned}
\frac{\partial \underline{V}(x, t)}{\partial t}= & d_{2} \mathcal{N}_{2}[\underline{V}(\cdot, t)](x) \\
& +\underline{V}(x, t)\left[\beta f(1)-r_{2}-\delta_{0}-\left(r_{2}+M\right) \underline{V}(x, t)\right], \quad x \in \mathbb{R}^{N}, t>\tau, \\
\underline{V}(x, 0) \leq & V(x, \tau) .
\end{aligned}\right.
$$

Then, because $\epsilon>0$ is small enough and Proposition 2.3(ii), we obtain that

$$
\liminf _{t \rightarrow \infty} \inf _{\|x\|<\left(c^{*}-\epsilon\right) t} V(x, t) \geq \liminf _{t \rightarrow \infty} \inf _{\|x\|<\left(c^{*}-\epsilon\right) t} \underline{V}(x, t)=\frac{\beta f(1)-r_{2}-\delta_{0}}{r_{2}+M}>0 .
$$

This completes the proof.

\section{Planar wave solutions and minimal wave speed}

In this section, we mainly investigate the planar wave solutions for system (1.1) connecting the predator-free state to a nontrivial state. In Section 4.1, we give a general result. We prove the existence of planar wave solutions for system (1.1) if $c \geq c^{*}$ in Section 4.2. In Section 4.3, we show the nonexistence of planar wave solutions for system (1.1) if $c \in\left(0, c^{*}\right)$. In Section 4.4, we explain that the spreading speed of the predatory in Theorem 3.1 is the minimal wave speed $c^{*}$.

\subsection{A general result}

In this subsection, we give a general result about the planar wave solutions of system (1.1) with (1.2). A solution $(U, V)$ to 1.1$)$ is called a planar wave solutions of (1.1), if there exists a function pair $(\Phi, \Psi)$, the wave profile, such that $(U, V)(x, t)=(\Phi, \Psi)(\xi), \xi:=\nu \cdot x+$ $c t$, where $c$ is a nonnegative constant, $\nu \in \mathbb{R}^{N}$ is a fixed unit vector and $x \cdot \nu=\sum_{j=1}^{N} x_{j} \nu_{j}$. Here, we focus on the planar waves connecting the predator-free state $(1,0)$ at $\xi=-\infty$ to a nontrivial state at $\xi=\infty$ in the sense that

$$
\liminf _{\xi \rightarrow \infty} \Phi(\xi)>0, \quad \liminf _{\xi \rightarrow \infty} \Psi(\xi)>0 .
$$


In fact, the "nontrivial state" at $\xi=\infty$ defined in the sense of (4.1) only means that the right tail of the planar wave is far from zero. It is difficult to describe the exact nontrivial state of the system due to the lack of comparison principle and the nonlocal diffusion of the system.

We make the following transformation:

$$
u(x, t)=1-U(x, t), \quad v(x, t)=V(x, t), \quad \bar{a}=\frac{a}{r_{1}} .
$$

With these notations, system $(1.1)$ is rewritten as

$$
\left\{\begin{array}{l}
\frac{\partial u}{\partial t}(x, t)=d_{1} \mathcal{N}_{1}[u(\cdot, t)](x)-r_{1} u(x, t)[1-u(x, t)]+f(1-u(x, t)) v(x, t), \\
\frac{\partial v}{\partial t}(x, t)=d_{2} \mathcal{N}_{2}[v(\cdot, t)](x)+r_{2} v(x, t)[\bar{\beta} f(1-u(x, t))-1-v(x, t)] .
\end{array}\right.
$$

At this time condition 1.3 becomes

$$
\bar{\beta} f(1)>1, \quad \bar{a}(\bar{\beta} f(1)-1)<1 .
$$

To find a planar wave solutions of 1.1 is equivalent to finding a solution for system 4.2 in the form

$$
u(x, t)=\phi(\xi), \quad v(x, t)=\psi(\xi), \quad \text { where } \xi:=\nu \cdot x+c t
$$

such that $(\phi, \psi)(-\infty)=(0,0)$ and

$$
\limsup _{\xi \rightarrow \infty} \phi(\xi)<1, \quad \liminf _{\xi \rightarrow \infty} \psi(\xi)>0 .
$$

Since $\nu$ is a unit vector, we can rotate the coordinates so that $\nu=(1,0, \ldots, 0)$ in the new coordinates. Due to the kernel function is a radial function, thus $(\phi(\xi), \psi(\xi))$ satisfies the following ordinary differential equation independently of the unit vector $\nu$ :

$$
\left\{\begin{array}{l}
c \phi^{\prime}(\xi)=d_{1} \widetilde{\mathcal{N}}_{1}[\phi](\xi)-r_{1} \phi(\xi)[1-\phi(\xi)]+f(1-\phi(\xi)) \psi(\xi), \\
c \psi^{\prime}(\xi)=d_{2} \widetilde{\mathcal{N}}_{2}[\psi](\xi)+r_{2} \psi(\xi)[\bar{\beta} f(1-\phi(\xi))-1-\psi(\xi)]
\end{array}\right.
$$

where the linear operators $\widetilde{\mathcal{N}}_{i}$ are defined by

$$
\tilde{\mathcal{N}}_{i}[\varphi](\xi):=\int_{\mathbb{R}} \widetilde{J}_{i}\left(x_{1}\right) \varphi\left(\xi-x_{1}\right) \mathrm{d} x_{1}-\varphi(\xi), \quad i=1,2,
$$

and

$$
\widetilde{J}_{i}\left(x_{1}\right):=\int_{\mathbb{R}^{N-1}} J_{i}\left(x_{1}, x_{2}, \ldots, x_{N}\right) \mathrm{d} x_{2} \cdots \mathrm{d} x_{N}, \quad i=1,2 .
$$

Thus, according to the definition of $\mathcal{N}_{i}, \widetilde{\mathcal{N}}_{i}(i=1,2)$ and 2.6 , we have

$$
c^{*}=\inf _{0<\lambda<\lambda_{0}} \frac{d_{2}\left[\int_{\mathbb{R}} \widetilde{J}_{2}\left(x_{1}\right) \mathrm{e}^{\lambda x_{1}} \mathrm{~d} x_{1}-1\right]+\beta f(1)-r_{2}}{\lambda} .
$$

We begin with the following definition. 
Definition 4.1. A pair of functions $(\bar{\phi}, \bar{\psi}),(\underline{\phi}, \underline{\psi}) \in X_{h}^{2}$ is called a pair of super and sub solutions of 4.4 if $(\underline{\phi}, \underline{\psi}) \leq(\bar{\phi}, \bar{\psi})$ for all $\xi \in \mathbb{R}$ and the following inequalities

$$
\begin{aligned}
& c \bar{\phi}^{\prime}(\xi) \geq d_{1} \widetilde{\mathcal{N}}_{1}[\bar{\phi}](\xi)-r_{1} \bar{\phi}(\xi)[1-\bar{\phi}(\xi)]+f(1-\bar{\phi}(\xi)) \bar{\psi}(\xi), \\
& c \bar{\psi}^{\prime}(\xi) \geq d_{2} \widetilde{\mathcal{N}}_{2}[\bar{\psi}](\xi)+r_{2} \bar{\psi}(\xi)[\bar{\beta} f(1-\underline{\phi}(\xi))-1-\bar{\psi}(\xi)], \\
& c \underline{\phi}^{\prime}(\xi) \leq d_{1} \widetilde{\mathcal{N}}_{1}[\phi](\xi)-r_{1} \underline{\phi}(\xi)[1-\underline{\phi}(\xi)]+f(1-\underline{\phi}(\xi)) \underline{\psi}(\xi), \\
& c \underline{\psi}^{\prime}(\xi) \leq d_{2} \tilde{\mathcal{N}}_{2}[\underline{\psi}](\xi)+r_{2} \underline{\psi}(\xi)[\bar{\beta} f(1-\bar{\phi}(\xi))-1-\underline{\psi}(\xi)]
\end{aligned}
$$

hold for all $\xi \in \mathbb{R} \backslash E$, where $E$ denotes some finite set $E \subset \mathbb{R}$ and $X_{h}^{2}:=X_{1} \times X_{\bar{\alpha}}$, where $\bar{\alpha}:=\bar{\beta} f(1)-1$.

Lemma 4.2. Let $c>0$ be given. Let $(\bar{\phi}, \bar{\psi}),(\underline{\phi}, \underline{\psi})$ be a pair of super and sub solutions of (4.4). Then, system (4.4) admits a unique solution $(\phi, \psi)$ such that

$$
(\underline{\phi}(\xi), \underline{\psi}(\xi)) \leq(\phi(\xi), \psi(\xi)) \leq(\bar{\phi}(\xi), \bar{\psi}(\xi)), \quad \xi \in \mathbb{R} .
$$

Proof. For each $(\phi, \psi) \in X_{h}^{2}$, we define the nonlinear operators $F_{1}, F_{2}$ on $X_{h}^{2}$ by

$$
\begin{aligned}
& F_{1}(\phi, \psi)(\xi):=M_{1} \phi(\xi)+d_{1} \widetilde{\mathcal{N}}_{1}[\phi](\xi)+f_{1}(\phi, \psi)(\xi), \\
& F_{2}(\phi, \psi)(\xi):=M_{1} \psi(\xi)+d_{2} \widetilde{\mathcal{N}}_{2}[\psi](\xi)+f_{2}(\phi, \psi)(\xi),
\end{aligned}
$$

where

$$
\begin{aligned}
& f_{1}(\phi, \psi)(\xi):=-r_{1} \phi(\xi)[1-\phi(\xi)]+f(1-\phi(\xi)) \psi(\xi), \\
& f_{2}(\phi, \psi)(\xi):=r_{2} \psi(\xi)[\bar{\beta} f(1-\phi(\xi))-1-\psi(\xi)] .
\end{aligned}
$$

Here, $M_{1}$ denotes some large positive constant such that $F_{1}$ and $F_{2}$ satisfy proper monotonicity in $\phi$ and $\psi$, respectively. With these notions, let $P=\left(P_{1}, P_{2}\right): X_{h}^{2} \rightarrow X^{2}$ be

$$
\begin{aligned}
& P_{1}(\phi, \psi)(\xi):=\frac{1}{c} \int_{-\infty}^{\xi} \mathrm{e}^{-\frac{M_{1}(\xi-s)}{c} F_{1}(\phi, \psi)(s) \mathrm{d} s} \\
& P_{2}(\phi, \psi)(\xi):=\frac{1}{c} \int_{-\infty}^{\xi} \mathrm{e}^{-\frac{M_{1}(\xi-s)}{c}} F_{2}(\phi, \psi)(s) \mathrm{d} s .
\end{aligned}
$$

Let $\mu>0$ be a small constant. Denote

$$
B_{\mu}\left(\mathbb{R}, \mathbb{R}^{2}\right):=\left\{(\phi, \psi) \in X^{2}:|(\phi, \psi)|_{\mu}:=\sup _{\xi \in \mathbb{R}} \max (|\phi(\xi)|,|\psi(\xi)|) e^{-\mu|\xi|}<\infty\right\} .
$$

Then, $\left(B_{\mu}\left(\mathbb{R}, \mathbb{R}^{2}\right),|\cdot|_{\mu}\right)$ is a Banach space. Define

$$
\Gamma:=\left\{(\phi, \psi) \in X_{h}^{2}:(\underline{\phi}, \underline{\psi}) \leq(\phi, \psi) \leq(\bar{\phi}, \bar{\psi})\right\} .
$$


Thus, $\Gamma$ is a nonempty convex, closed and bounded with respect to the norm $|\cdot|_{\mu}$.

Since $f_{1}(\phi, \psi)$ is monotone increasing in $\psi$ and $f_{2}(\phi, \psi)$ is monotone decreasing in $\phi$, we have

$$
\begin{aligned}
& F_{1}(\phi, \psi)(\xi) \geq M_{1} \underline{\phi}(\xi)+d_{1} \tilde{\mathcal{N}}_{1}[\phi](\xi)+f_{1}(\underline{\phi}, \underline{\psi})(\xi)=: \underline{F}_{1}, \\
& F_{1}(\phi, \psi)(\xi) \leq M_{1} \bar{\phi}(\xi)+d_{1} \widetilde{\mathcal{N}}_{1}[\bar{\phi}](\xi)+f_{1}(\bar{\phi}, \bar{\psi})(\xi)=: \bar{F}_{1}, \\
& F_{2}(\phi, \psi)(\xi) \geq M_{1} \underline{\psi}(\xi)+d_{2} \widetilde{\mathcal{N}}_{2}[\underline{\psi}](\xi)+f_{2}(\bar{\phi}, \underline{\psi})(\xi)=: \underline{F}_{2}, \\
& F_{2}(\phi, \psi)(\xi) \leq M_{1} \bar{\psi}(\xi)+d_{2} \widetilde{\mathcal{N}}_{2}[\bar{\psi}](\xi)+f_{2}(\underline{\phi}, \bar{\psi})(\xi)=: \bar{F}_{2} .
\end{aligned}
$$

Let

$$
\underline{P}_{1}(\xi):=\frac{1}{c} \int_{-\infty}^{\xi} \mathrm{e}^{-\frac{M_{1}(\xi-s)}{c}} \underline{F}_{1}(s) \mathrm{d} s, \quad \bar{P}_{1}(\xi):=\frac{1}{c} \int_{-\infty}^{\xi} \mathrm{e}^{-\frac{M_{1}(\xi-s)}{c}} \bar{F}_{1}(s) \mathrm{d} s, \quad \xi \in \mathbb{R} .
$$

Then it suffices to prove that

$$
\underline{\phi}(\xi) \leq \underline{P}_{1}(\xi) \leq \bar{P}_{1}(\xi) \leq \bar{\phi}(\xi), \quad \xi \in \mathbb{R} .
$$

Indeed, let

$$
E=\left\{E_{1}, E_{2}, \ldots, E_{n}\right\}
$$

and denote $E_{0}=-\infty, E_{n+1}=\infty$. If $\xi \in\left(E_{k-1}, E_{k}\right)$ with some $k \in\{1,2, \ldots, n+1\}$, then

$$
\begin{aligned}
\bar{P}_{1}(\xi) & =\frac{1}{c} \int_{-\infty}^{\xi} \mathrm{e}^{-\frac{M_{1}(\xi-s)}{c}} \bar{F}_{1}(s) \mathrm{d} s \\
& =\left(\sum_{i=0}^{k-1} \frac{1}{c} \int_{E_{i-1}}^{E_{i}}+\frac{1}{c} \int_{E_{i-1}}^{\xi}\right) \mathrm{e}^{-\frac{M_{1}(\xi-s)}{c}} \bar{F}_{1}(s) \mathrm{d} s \\
& \leq\left(\sum_{i=0}^{k-1} \frac{1}{c} \int_{E_{i-1}}^{E_{i}}+\frac{1}{c} \int_{E_{i-1}}^{\xi}\right) \mathrm{e}^{-\frac{M_{1}(\xi-s)}{c}}\left(c \bar{\phi}^{\prime}(s)+M_{1} \bar{\phi}(s)\right) \mathrm{d} s \\
& =\bar{\phi}(\xi) .
\end{aligned}
$$

Since $\bar{P}_{1}(\xi)$ and $\bar{\phi}(\xi)$ are continuous functions, we obtain

$$
\bar{P}_{1}(\xi) \leq \bar{\phi}(\xi), \quad \xi \in \mathbb{R}
$$

In a similar way, we can deduce that

$$
\underline{\phi}(\xi) \leq \underline{P}_{1}(\xi), \quad \xi \in \mathbb{R}
$$

and

$$
\underline{\psi}(\xi) \leq P_{2}(\phi, \psi)(\xi) \leq \bar{\psi}(\xi), \quad \xi \in \mathbb{R} .
$$

Thus, we have $P(\Gamma) \subset \Gamma$. In addition, if $\mu$ satisfies $\mu<\frac{M_{1}}{2 c}$ (the proof is similar to 16 , Lemma 3.4]), we obtain that $P: \Gamma \rightarrow \Gamma$ is complete continuous in the sense of $|\cdot|_{\mu}$. Thus, using Schauder's fixed point theorem, we complete the proof of Lemma 4.2 . 
4.2. Existence of planar wave solutions for $c \geq c^{*}$

In order to construct a pair of proper super-sub solutions, we consider the function

$$
\begin{aligned}
\Delta(\lambda, c) & :=d_{2}\left[\int_{\mathbb{R}^{N}} J_{2}(x) e^{\lambda x_{1}} \mathrm{~d} x-1\right]-c \lambda+r_{2}(\bar{\beta} f(1)-1) \\
& =d_{2}\left[\int_{\mathbb{R}} \widetilde{J}_{2}\left(x_{1}\right) e^{\lambda x_{1}} \mathrm{~d} x_{1}-1\right]-c \lambda+r_{2}(\bar{\beta} f(1)-1),
\end{aligned}
$$

where $\bar{\beta}:=\frac{\beta}{r_{2}}$. By the convexity, we have the following conclusion.

Lemma 4.3. Let Assumption 1.1 be satisfied. Then the following statements hold.

(i) If $c>c^{*}$, then the equation $\Delta(\lambda, c)=0$ has two positive roots $\lambda_{1}(c), \lambda_{2}(c)$, and $0<\lambda_{1}(c)<\lambda_{2}(c)<\lambda_{0}$. Moreover, $\Delta(\lambda, c)<0$, if $\lambda \in\left(\lambda_{1}(c), \lambda_{2}(c)\right) . \Delta(\lambda, c)>0$, if $\lambda \in\left(0, \lambda_{1}(c)\right) \cup\left(\lambda_{2}(c), \lambda_{0}\right)$.

(ii) If $0<c<c^{*}$, then $\Delta(\lambda, c)>0$ for all $\lambda \in\left(0, \lambda_{0}\right)$.

(iii) If $c=c^{*}>0$, then $\Delta\left(\lambda, c^{*}\right) \geq 0$ for any $\lambda \in\left(0, \lambda_{0}\right)$ and $\Delta\left(\lambda, c^{*}\right)=0$ has a unique $\operatorname{root} \lambda^{*}$.

The following theorem presents us the existence of nonnegative solutions of system (4.4) with $c>c^{*}$ connecting the predator-free equilibrium $(1,0)$ at $\xi=-\infty$.

Theorem 4.4. Let $c>c^{*}$ be given and fixed. Then, (4.4) admits a nonnegative solution $(\phi, \psi)$ such that $\lim \sup _{\xi \rightarrow-\infty}(\phi(\xi), \psi(\xi))=(0,0)$.

Proof. Let $c>c^{*}$ be given and fixed. We define the following continuous functions:

$$
\begin{gathered}
\bar{\phi}(\xi)=\left\{\begin{array}{ll}
K \mathrm{e}^{\lambda \xi}, & \xi \leq \xi_{0}, \\
1, & \xi \geq \xi_{0},
\end{array} \quad \bar{\psi}(\xi)= \begin{cases}\mathrm{e}^{\lambda_{1} \xi}, & \xi \leq \xi_{1}, \\
\bar{\beta} f(1)-1, & \xi \geq \xi_{1},\end{cases} \right. \\
\underline{\phi}(\xi) \equiv 0, \quad \underline{\psi}(\xi)= \begin{cases}\mathrm{e}^{\lambda_{1} \xi}-q \mathrm{e}^{\eta \lambda_{1} \xi}, & \xi \leq \xi_{2}, \\
0, & \xi \geq \xi_{2} .\end{cases}
\end{gathered}
$$

Define

$$
A(\lambda):=d_{1}\left[\int_{\mathbb{R}} \widetilde{J}_{1}\left(x_{1}\right) \mathrm{e}^{\lambda x_{1}} \mathrm{~d} x_{1}-1\right]-c \lambda .
$$

Since $A(0)=0$ and

$$
A^{\prime}(0)=\lim _{\lambda \rightarrow 0}\left\{d_{1} \int_{\mathbb{R}} \widetilde{J}_{1}\left(x_{1}\right) x_{1} \mathrm{e}^{\lambda x_{1}} \mathrm{~d} x_{1}-c\right\}=-c<0,
$$

there exists $\lambda \in\left(0, \min \left\{\lambda_{0}, \lambda_{1}(c)\right\}\right)$ such that

$$
A(\lambda)=d_{1}\left[\int_{\mathbb{R}} \widetilde{J}_{1}\left(x_{1}\right) \mathrm{e}^{\lambda x_{1}} \mathrm{~d} x_{1}-1\right]-c \lambda<0 .
$$


For notational simplicity, since $c$ is fixed in this proof, we simply denote $\lambda_{i}=\lambda_{i}(c), i=1,2$.

First, we define $\xi_{1}$ by $\mathrm{e}^{\lambda_{1} \xi_{1}}=\bar{\beta} f(1)-1$ and choose a constant $\xi_{0}<\min \left\{0, \xi_{1}\right\}$ such that $\bar{a} \mathrm{e}^{\left(\lambda_{1}-\lambda\right) \xi_{0}}<1$. Next, we set $K=\mathrm{e}^{-\lambda \xi_{0}}$. Clearly $K>1$ and

$$
\bar{a} \mathrm{e}^{\lambda_{1} \xi}<\mathrm{e}^{\lambda \xi}, \quad \forall \xi<\xi_{0} .
$$

Then, take $\eta \in(1,2)$ such that

$$
\eta \lambda_{1}<\min \left\{\lambda_{2}, \lambda_{1}+\lambda\right\}
$$

Finally, for $q>1$ define $\xi_{2}=\xi_{2}(q)<0$ by $\mathrm{e}^{(\eta-1) \lambda_{1} \xi_{2}}=\frac{1}{q}$. Letting $q \rightarrow \infty$, we have $\xi_{2} \rightarrow-\infty$. We can take a constant $q>1$ large enough such that $\xi_{2} \leq \xi_{0}$ and

$$
q>\frac{r_{2}+r_{2} \bar{\beta} f(1) K}{-\triangle\left(\eta \lambda_{1}, c\right)}+1 .
$$

Obviously, $\underline{\phi}(\xi) \leq \bar{\phi}(\xi)$ for any $\xi \in \mathbb{R}$. Since $\xi_{2}<\xi_{1}$, we also have $\underline{\psi}(\xi) \leq \bar{\psi}(\xi)$ for all $\xi \in \mathbb{R}$.

Now we prove that the functions $(\bar{\phi}, \bar{\psi})$ and $(\underline{\phi}, \underline{\psi})$ are a pair of super and sub solutions of (4.4) with $(\bar{\phi}, \bar{\psi})(-\infty)=(\underline{\phi}, \underline{\psi})(-\infty)=(0,0)$.

For $\xi>\xi_{0}$, we have $\bar{\phi}(\xi)=1$ and $\bar{\phi}^{\prime}(\xi)=0$, so that

$$
\begin{aligned}
& d_{1} \widetilde{\mathcal{N}}_{1}[\bar{\phi}](\xi)-r_{1} \bar{\phi}(\xi)[1-\bar{\phi}(\xi)]+f(1-\bar{\phi}(\xi)) \bar{\psi}(\xi)-c \bar{\phi}^{\prime}(\xi) \\
= & d_{1}\left[\int_{\mathbb{R}} \widetilde{J}_{1}\left(x_{1}\right) \bar{\phi}\left(\xi-x_{1}\right) \mathrm{d} x_{1}-\bar{\phi}(\xi)\right] \leq 0 .
\end{aligned}
$$

Thus, condition 4.5 holds for $\xi>\xi_{0}$.

For $\xi<\xi_{0}$, we have $\bar{\phi}(\xi)=K \mathrm{e}^{\lambda \xi}<1$. Moreover, $\bar{\phi}(\xi) \leq K \mathrm{e}^{\lambda \xi}$ for all $\xi \in \mathbb{R}$ and $\bar{\psi}(\xi)=\mathrm{e}^{\lambda_{1} \xi}$ for all $\xi<\xi_{0} \leq \xi_{1}$. Using $f(x)<a x$ and the symmetry of the function $\widetilde{J}_{1}$, it follows from 4.9 and 4.10 that

$$
\begin{aligned}
& d_{1} \widetilde{\mathcal{N}}_{1}[\bar{\phi}](\xi)+f(1-\bar{\phi}(\xi)) \bar{\psi}(\xi)-r_{1} \bar{\phi}(\xi)[1-\bar{\phi}(\xi)]-c \bar{\phi}^{\prime}(\xi) \\
\leq & d_{1}\left[\int_{\mathbb{R}} \widetilde{J}_{1}\left(x_{1}\right) \bar{\phi}\left(\xi-x_{1}\right) \mathrm{d} x_{1}-K \mathrm{e}^{\lambda \xi}\right]+a(1-\bar{\phi}(\xi)) \bar{\psi}(\xi)-r_{1} \bar{\phi}(\xi)\left[1-K \mathrm{e}^{\lambda \xi}\right]-c \lambda K \mathrm{e}^{\lambda \xi} \\
\leq & d_{1}\left[\int_{\mathbb{R}} K \widetilde{J}_{1}\left(x_{1}\right) \mathrm{e}^{\lambda\left(\xi-x_{1}\right)} \mathrm{d} x_{1}-K \mathrm{e}^{\lambda \xi}\right]+r_{1}\left(1-K \mathrm{e}^{\lambda \xi}\right)\left[\frac{a}{r_{1}} \mathrm{e}^{\lambda_{1} \xi}-K \mathrm{e}^{\lambda \xi}\right]-c \lambda K \mathrm{e}^{\lambda \xi} \\
\leq & K \mathrm{e}^{\lambda \xi}\left\{d_{1}\left[\int_{\mathbb{R}} \widetilde{J}_{1}\left(x_{1}\right) \mathrm{e}^{\lambda x_{1}} \mathrm{~d} x_{1}-1\right]-c \lambda\right\} \leq 0 .
\end{aligned}
$$

Hence, condition (4.5) also holds for $\xi<\xi_{0}$ and thus for any $\xi \neq \xi_{0}$.

We now consider 4.6). For $\xi>\xi_{1}$, we get $\bar{\psi}(\xi)=\bar{\beta} f(1)-1$. Since $\bar{\psi}(\xi) \leq \bar{\beta} f(1)-1$ for all $\xi \in \mathbb{R}$, it follows that

$$
d_{2} \widetilde{\mathcal{N}}_{2}[\bar{\psi}](\xi)+r_{2} \bar{\psi}(\xi)[\bar{\beta} f(1-\underline{\phi}(\xi))-1-\bar{\psi}(\xi)]-c \bar{\psi}^{\prime}(\xi)
$$




$$
\begin{aligned}
& \leq d_{2}\left[\int_{\mathbb{R}}(\bar{\beta} f(1)-1) \widetilde{J}_{2}\left(x_{1}\right) \mathrm{d} x_{1}-(\bar{\beta} f(1)-1)\right]+r_{2}(\bar{\beta} f(1)-1)[\bar{\beta} f(1)-1-(\bar{\beta} f(1)-1)] \\
& =0
\end{aligned}
$$

Hence, (4.6) holds for $\xi>\xi_{1}$.

For $\xi<\xi_{1}$, we get $\bar{\psi}(\xi)=\mathrm{e}^{\lambda_{1} \xi}$. Applying $\bar{\psi}(\xi) \leq \mathrm{e}^{\lambda_{1} \xi}$ for all $\xi \in \mathbb{R}$ and $\underline{\phi}(\xi) \equiv 0$, we deduce that

$$
\begin{aligned}
& d_{2} \widetilde{\mathcal{N}}_{2}[\bar{\psi}](\xi)-c \bar{\psi}^{\prime}(\xi)+r_{2} \bar{\psi}(\xi)[\bar{\beta} f(1-\underline{\phi}(\xi))-1-\bar{\psi}(\xi)] \\
= & d_{2} \widetilde{\mathcal{N}}_{2}[\bar{\psi}](\xi)-c \bar{\psi}^{\prime}(\xi)+r_{2} \bar{\psi}(\xi)[\bar{\beta} f(1)-1-\bar{\psi}(\xi)] \\
\leq & d_{2} \widetilde{\mathcal{N}}_{2}[\bar{\psi}](\xi)-c \bar{\psi}^{\prime}(\xi)+r_{2}(\bar{\beta} f(1)-1) \bar{\psi}(\xi) \\
= & d_{2}\left[\int_{\mathbb{R}} \bar{\psi}\left(\xi-x_{1}\right) \widetilde{J}_{2}\left(x_{1}\right) \mathrm{d} x_{1}-\mathrm{e}^{\lambda_{1} \xi}\right]-c \lambda_{1} \mathrm{e}^{\lambda_{1} \xi}+r_{2}(\bar{\beta} f(1)-1) \mathrm{e}^{\lambda_{1} \xi} \\
\leq & \mathrm{e}^{\lambda_{1} \xi} \triangle\left(\lambda_{1}, c\right)=0
\end{aligned}
$$

for all $\xi<\xi_{1}$. Hence, (4.6) also holds for $\xi<\xi_{1}$ and thus for all $\xi \in \mathbb{R} \backslash\left\{\xi_{1}\right\}$.

Now consider 4.7 . Since $\underline{\phi}(\xi) \equiv 0$, using $(\mathrm{H} 1)$ and $\underline{\psi}(\xi) \geq 0$, it follows that

$$
d_{1} \widetilde{\mathcal{N}}_{1}[\underline{\phi}](\xi)-r_{1} \underline{\phi}(\xi)[1-\underline{\phi}(\xi)]+f(1-\underline{\phi}(\xi)) \underline{\psi}(\xi)-c \underline{\phi^{\prime}}(\xi)=f(1) \underline{\psi}(\xi) \geq 0 .
$$

Hence, 4.7) holds for all $\xi \in \mathbb{R}$. It remains to check that 4.8 holds true. Clearly, for $\xi>\xi_{2}$, we have $\underline{\psi}(\xi)=0$ and 4.8 holds true for $\xi>\xi_{2}$.

For $\xi<\xi_{2}$, one has $\underline{\psi}(\xi)=\mathrm{e}^{\lambda_{1} \xi}-q \mathrm{e}^{\eta \lambda_{1} \xi}$. Since $\xi_{2} \leq \xi_{0}$, we have $\bar{\phi}(\xi)=K \mathrm{e}^{\lambda \xi}$, thus $0<\bar{\phi}(\xi)<1$. In addition, for any $\xi \in \mathbb{R}$, one has $\underline{\psi}(\xi) \leq \mathrm{e}^{\lambda_{1} \xi}$ and $\underline{\psi}(\xi) \geq \mathrm{e}^{\lambda_{1} \xi}-q \mathrm{e}^{\eta \lambda_{1} \xi}$. Using (H3), $\xi<\xi_{2}<0$ and $\triangle\left(\lambda_{1}, c\right)=0$, from 4.11) and 4.12), it follows that

$$
\begin{aligned}
& d_{2} \widetilde{\mathcal{N}}_{2}[\psi](\xi)+r_{2} \underline{\psi}(\xi)[\bar{\beta} f(1-\bar{\phi}(\xi))-1-\underline{\psi}(\xi)]-c \underline{\psi}^{\prime}(\xi) \\
\geq & d_{2}\left[\int_{\mathbb{R}} \widetilde{J}_{2}\left(x_{1}\right) \underline{\psi}\left(\xi-x_{1}\right) \mathrm{d} x_{1}-\left(\mathrm{e}^{\lambda_{1} \xi}-q \mathrm{e}^{\eta \lambda_{1} \xi}\right)\right] \\
& +r_{2} \underline{\psi}(\xi)[\bar{\beta} f(1)(1-\bar{\phi}(\xi))-1-\underline{\psi}(\xi)]-c\left(\lambda_{1} \mathrm{e}^{\lambda_{1} \xi}-q \lambda_{1} \eta \mathrm{e}^{\eta \lambda_{1} \xi}\right) \\
= & d_{2}\left[\int_{\mathbb{R}} \widetilde{J}_{2}\left(x_{1}\right) \underline{\psi}\left(\xi-x_{1}\right) \mathrm{d} x_{1}-\left(\mathrm{e}^{\lambda_{1} \xi}-q \mathrm{e}^{\eta \lambda_{1} \xi}\right)\right] \\
& +r_{2} \underline{\psi}(\xi)(\bar{\beta} f(1)-1)-r_{2} \bar{\beta} f(1) \underline{\psi}(\xi) \bar{\phi}(\xi)-r_{2} \underline{\psi}^{2}(\xi)-c\left(\lambda_{1} \mathrm{e}^{\lambda_{1} \xi}-q \lambda_{1} \eta \mathrm{e}^{\eta \lambda_{1} \xi}\right) \\
\geq & d_{2}\left[\int_{\mathbb{R}} \widetilde{J}_{2}\left(x_{1}\right)\left(\mathrm{e}^{\lambda_{1}\left(\xi-x_{1}\right)}-q \mathrm{e}^{\eta \lambda_{1}\left(\xi-x_{1}\right)}\right) \mathrm{d} x_{1}-\left(\mathrm{e}^{\lambda_{1} \xi}-q \mathrm{e}^{\eta \lambda_{1} \xi}\right)\right] \\
& +r_{2}(\bar{\beta} f(1)-1)\left(\mathrm{e}^{\lambda_{1} \xi}-q \mathrm{e}^{\eta \lambda_{1} \xi}\right)-r_{2} \bar{\beta} f(1) K \mathrm{e}^{\left(\lambda+\lambda_{1}\right) \xi}-r_{2} \mathrm{e}^{2 \lambda_{1} \xi}-c\left(\lambda_{1} \mathrm{e}^{\lambda_{1} \xi}-q \lambda_{1} \eta \mathrm{e}^{\eta \lambda_{1} \xi}\right) \\
\geq & \mathrm{e}^{\lambda_{1} \xi} \triangle\left(\lambda_{1}, c\right)+\mathrm{e}^{\eta \lambda_{1} \xi}\left\{-q \triangle\left(\eta \lambda_{1}, c\right)-r_{2} \mathrm{e}^{(2-\eta) \lambda_{1} \xi}-r_{2} \bar{\beta} f(1) K \mathrm{e}^{\left(\lambda+\lambda_{1}-\eta \lambda_{1}\right) \xi}\right\} \\
\geq & \mathrm{e}^{\eta \lambda_{1} \xi}\left[-q \triangle\left(\eta \lambda_{1}, c\right)-r_{2}-r_{2} \bar{\beta} f(1) K\right]>0 .
\end{aligned}
$$


Then we get that (4.8) also holds for $\xi<\xi_{2}$ and thus for any $\xi \in \mathbb{R} \backslash\left\{\xi_{2}\right\}$.

It follows from Lemma 4.2 that there exists a unique solution $(\phi, \psi)$ with $(\underline{\phi}, \underline{\psi}) \leq$ $(\phi, \psi) \leq(\bar{\phi}, \bar{\psi})$. Since $(\bar{\phi}, \bar{\psi})(-\infty)=(\underline{\phi}, \underline{\psi})(-\infty)=(0,0)$, we conclude that $(\phi, \psi)(-\infty)=$ $(0,0)$. This is the end.

We now derive the existence of nonnegative solutions of system 4.4 with $c=c^{*}$ connecting the predator-free equilibrium $(1,0)$ at $\xi=-\infty$.

Theorem 4.5. Assume that the function $J_{2}$ is compactly supported. Then, system (4.4) with $c=c^{*}$ admits a nonnegative solution $(\phi, \psi)$ such that $\lim _{\xi \rightarrow-\infty}(\phi(\xi), \psi(\xi))=(0,0)$.

Proof. The approach is to construct a suitable pair of super and sub solutions satisfying the limit condition $(\bar{\phi}, \bar{\psi})(-\infty)=(\underline{\phi}, \underline{\psi})(-\infty)=(0,0)$.

We set the following functions:

$$
\begin{gathered}
\bar{\phi}(\xi)=\left\{\begin{array}{ll}
K \mathrm{e}^{\lambda \xi}, & \xi \leq \xi_{0}, \\
1, & \xi \geq \xi_{0},
\end{array} \quad \bar{\psi}(\xi)= \begin{cases}-L \xi \mathrm{e}^{\lambda^{*} \xi}, & \xi \leq \xi_{1}, \\
\bar{\beta} f(1)-1, & \xi \geq \xi_{1},\end{cases} \right. \\
\underline{\phi}(\xi) \equiv 0, \quad \underline{\psi}(\xi)= \begin{cases}(-L \xi-q \sqrt{-\xi}) \mathrm{e}^{\lambda^{*} \xi}, & \xi \leq \xi_{3}, \\
0, & \xi \geq \xi_{3},\end{cases}
\end{gathered}
$$

where $L, K, q$ are constants to be determined (in order) later while the constant $\lambda \in$ $\left(0, \min \left\{\lambda_{0}, \lambda^{*} / 2\right\}\right)$ is defined as before so that 4.9$)$ holds with $c=c^{*}$.

Since $J_{2}$ has a compact support, we choose a positive constant $\mathrm{G}$ such that

$$
J_{2}(x)=0, \quad\|x\|>G .
$$

For any large enough constant $L$, the following equation $-L \xi \mathrm{e}^{\lambda^{*} \xi}=\bar{\beta} f(1)-1$ has two negative roots, denoted by $\xi_{1}, \xi_{2}$ and $\xi_{1}<\xi_{2}$, and

$$
\xi_{2}-\xi_{1}>G
$$

It is easy to see that

$$
\xi_{1}<-\frac{1}{\lambda^{*}}<\xi_{2}<0 \text { and } L \xi \mathrm{e}^{\lambda^{*} \xi}>\bar{\beta} f(1)-1 \quad \text { for all } \xi \in\left(\xi_{1}, \xi_{2}\right) .
$$

Fix $\lambda_{*} \in\left(\lambda^{*}-\lambda, \lambda^{*}\right)$. Since $\lambda<\lambda^{*} / 2$, we have $\lambda_{*}>\lambda$. Because $\lambda_{*}-\lambda^{*}-\lambda<0$, we can take $\xi_{0}<0$ such that

$$
\xi_{0}<\min \left\{-1 /\left(\lambda^{*}-\lambda_{*}\right), \xi_{1}\right\}, \quad \mathrm{e}^{\left(\lambda_{*}-\lambda^{*}-\lambda\right) \xi_{0}} /\left(-\xi_{0}\right)>\bar{a} L .
$$

For such a fixed $\xi_{0}$, we set $K:=\mathrm{e}^{-\lambda \xi_{0}}$. Define function $s: \mathbb{R} \rightarrow \mathbb{R}$ as $s(\xi):=(-\xi) \mathrm{e}^{\left(\lambda^{*}-\lambda_{*}\right) \xi}$. It easy to see that $s(\xi)$ is increasing on $\left(-\infty,-1 /\left(\lambda^{*}-\lambda_{*}\right)\right)$. Then, we have

$$
s(\xi)<s\left(\xi_{0}\right) \text { for } \xi \leq \xi_{0},
$$


hence, it follows that

$$
-\bar{a} L \xi \mathrm{e}^{\left(\lambda^{*}-\lambda_{*}\right) \xi}<-\bar{a} L \xi_{0} \mathrm{e}^{\left(\lambda^{*}-\lambda_{*}\right) \xi_{0}} .
$$

Since $\lambda_{*}>\lambda$ and (4.14), we deduce that

$$
-\bar{a} L \xi \mathrm{e}^{\lambda^{*} \xi}<K \mathrm{e}^{\lambda_{*} \xi}<K \mathrm{e}^{\lambda \xi} \text { for all } \xi \leq \xi_{0} .
$$

According to 2, 6], we know that the function $z \mapsto Y(z):=\left[-L z-q(-z)^{1 / 2}\right] \mathrm{e}^{\lambda^{*} z}$ is positive and has a unique critical (maximal) point in $\left(-\infty, \xi_{3}\right)$, where $\xi_{3}:=-(q / L)^{2}$, for any $q>0$. Then, we take $q$ large enough such that

$$
\begin{gathered}
\xi_{3}<\xi_{0}, \\
q \geq \frac{\max _{\xi<0}\left\{8(-\xi+G)^{3 / 2}\left[r_{2} \bar{\beta} f(1) \frac{K^{2}}{\bar{a}} \mathrm{e}^{\left(\lambda+\lambda_{*}-\lambda^{*}\right) \xi}+r_{2} \frac{K^{2}}{\bar{a}^{2}} \mathrm{e}^{\left(2 \lambda_{*}-\lambda^{*}\right) \xi}\right]\right\}}{d_{2} \int_{\mathbb{R}} \widetilde{J}_{2}\left(x_{1}\right) x_{1}^{2} \mathrm{e}^{-\lambda^{*} x_{1}} \mathrm{~d} x_{1}} .
\end{gathered}
$$

It is clear that $\underline{\phi} \leq \bar{\phi}$ on $\mathbb{R}$, and $\underline{\psi}<\bar{\psi}$ on $\mathbb{R}$ by 4.16 ), $\xi_{3}<\xi_{0}<\xi_{1}$. Clearly (4.5) holds for $\xi>\xi_{0}$.

For $\xi<\xi_{0}$, one has $\bar{\phi}(\xi)=K \mathrm{e}^{\lambda \xi}<1$. Since $f(x) \leq a x$ and $\bar{\phi}(\xi) \leq K \mathrm{e}^{\lambda \xi}$ for all $\xi \in \mathbb{R}$. Using $\xi_{0}<\xi_{1}, 4.9$ and 4.15 , it follows that

$$
\begin{aligned}
& d_{1} \widetilde{\mathcal{N}}_{1}[\bar{\phi}](\xi)+f(1-\bar{\phi}(\xi)) \bar{\psi}(\xi)-r_{1} \bar{\phi}(\xi)[1-\bar{\phi}(\xi)]-c^{*} \bar{\phi}^{\prime}(\xi) \\
\leq & d_{1}\left[\int_{\mathbb{R}} \widetilde{J}_{1}\left(x_{1}\right) \bar{\phi}\left(\xi-x_{1}\right) \mathrm{d} x_{1}-K \mathrm{e}^{\lambda \xi}\right]+a(1-\bar{\phi}(\xi)) \bar{\psi}(\xi)-r_{1} \bar{\phi}(\xi)\left(1-K \mathrm{e}^{\lambda \xi}\right)-c^{*} \lambda K \mathrm{e}^{\lambda \xi} \\
\leq & K \mathrm{e}^{\lambda \xi}\left\{d_{1}\left[\int_{\mathbb{R}} \widetilde{J}_{1}\left(x_{1}\right) \mathrm{e}^{\lambda x_{1}} \mathrm{~d} x_{1}-1\right]-c^{*} \lambda\right\}+r_{1}\left(1-K \mathrm{e}^{\lambda \xi}\right)\left[\frac{-a}{r_{1}} L \xi \mathrm{e}^{\lambda^{*} \xi}-K \mathrm{e}^{\lambda \xi}\right]<0
\end{aligned}
$$

for $\xi<\xi_{0}$. Hence, condition (4.5) also holds for $\xi<\xi_{0}$ and thus for any $\xi \neq \xi_{0}$.

We now turn to 4.6). For $\xi>\xi_{1}$, we have $\bar{\psi}(\xi)=\bar{\beta} f(1)-1$. Since $\bar{\psi}(\xi) \leq \bar{\beta} f(1)-1$ for all $\xi \in \mathbb{R}$, we have

$$
\begin{aligned}
& d_{2} \widetilde{\mathcal{N}}_{2}[\bar{\psi}](\xi)+r_{2} \bar{\psi}(\xi)[\bar{\beta} f(1-\underline{\phi}(\xi))-1-\bar{\psi}(\xi)]-c \bar{\psi}^{\prime}(\xi) \\
\leq & d_{2}\left[\int_{\mathbb{R}}(\bar{\beta} f(1)-1) \widetilde{J}_{2}\left(x_{1}\right) \mathrm{d} x_{1}-(\bar{\beta} f(1)-1)\right] \\
& +r_{2}(\bar{\beta} f(1)-1)[\bar{\beta} f(1)-1-(\bar{\beta} f(1)-1)]=0 .
\end{aligned}
$$

Hence, (4.6) holds for $\xi>\xi_{1}$.

For $\xi<\xi_{1}$, from the assumption that $J_{2}$ has a compact support in $\bar{B}_{G}(0)$ and (4.13), we obtain $\xi-x_{1} \leq \xi_{1}+G<\xi_{2}$ for all $x_{1} \in[-G, G]$ and

$$
\begin{aligned}
\int_{\mathbb{R}} \bar{\psi}\left(\xi-x_{1}\right) \widetilde{J}_{2}\left(x_{1}\right) \mathrm{d} x_{1} & =\int_{-G}^{G} \bar{\psi}\left(\xi-x_{1}\right) \widetilde{J}_{2}\left(x_{1}\right) \mathrm{d} x_{1} \\
& \leq \int_{\mathbb{R}}\left\{-L\left(\xi-x_{1}\right) \mathrm{e}^{\lambda^{*}\left(\xi-x_{1}\right)}\right\} \widetilde{J}_{2}\left(x_{1}\right) \mathrm{d} x_{1} .
\end{aligned}
$$


Hence, we have

$$
\begin{aligned}
& d_{2} \widetilde{\mathcal{N}}_{2}[\bar{\psi}](\xi)-c^{*} \bar{\psi}^{\prime}(\xi)+r_{2} \bar{\psi}(\xi)[\bar{\beta} f(1-\underline{\phi}(\xi))-1-\bar{\psi}(\xi)] \\
= & d_{2} \widetilde{\mathcal{N}}_{2}[\bar{\psi}](\xi)-c^{*} \bar{\psi}^{\prime}(\xi)+r_{2} \bar{\psi}(\xi)[\bar{\beta} f(1)-1-\bar{\psi}(\xi)] \\
\leq & d_{2} \widetilde{\mathcal{N}}_{2}[\bar{\psi}](\xi)-c^{*} \bar{\psi}^{\prime}(\xi)+r_{2}(\bar{\beta} f(1)-1) \bar{\psi}(\xi) \\
= & d_{2}\left[\int_{\mathbb{R}} \bar{\psi}\left(\xi-x_{1}\right) \widetilde{J}_{2}\left(x_{1}\right) \mathrm{d} x_{1}+L \xi \mathrm{e}^{\lambda^{*} \xi}\right]+c^{*} L\left(\lambda^{*} \xi+1\right) \mathrm{e}^{\lambda^{*} \xi}-r_{2} L(\bar{\beta} f(1)-1) \xi \mathrm{e}^{\lambda^{*} \xi} \\
\leq & d_{2}\left[\int_{\mathbb{R}}\left\{-L\left(\xi-x_{1}\right) \mathrm{e}^{\lambda^{*}\left(\xi-x_{1}\right)}\right\} \widetilde{J}_{2}\left(x_{1}\right) \mathrm{d} x_{1}+L \xi \mathrm{e}^{\lambda^{*} \xi}\right] \\
& +c^{*} L\left(\lambda^{*} \xi+1\right) \mathrm{e}^{\lambda^{*} \xi}-r_{2} L(\bar{\beta} f(1)-1) \xi \mathrm{e}^{\lambda^{*} \xi} \\
= & -L \xi \mathrm{e}^{\lambda^{*} \xi}\left\{d_{2}\left[\int_{\mathbb{R}} \mathrm{e}^{\lambda^{*} x_{1}} \widetilde{J}_{2}\left(x_{1}\right) \mathrm{d} x_{1}-1\right]-c^{*} \lambda^{*}+r_{2}(\bar{\beta} f(1)-1)\right\} \\
& +L \mathrm{e}^{\lambda^{*} \xi}\left[d_{2} \int_{\mathbb{R}} x_{1} \mathrm{e}^{-\lambda^{*} x_{1}} \widetilde{J}_{2}\left(x_{1}\right) \mathrm{d} x_{1}+c^{*}\right]=0
\end{aligned}
$$

for all $\xi<\xi_{1}$, where we use a change of variable $z=-x_{1}$ yielding

$$
d_{2} \int_{-\infty}^{+\infty} x_{1} \mathrm{e}^{-\lambda^{*} x_{1}} \widetilde{J}_{2}\left(x_{1}\right) d x_{1}=-d_{2} \int_{-\infty}^{+\infty} z \mathrm{e}^{\lambda^{*} z} \widetilde{J}_{2}(z) d z=-c^{*} .
$$

Hence, 4.6 also holds for $\xi<\xi_{1}$ and thus for all $\xi \in \mathbb{R} \backslash\left\{\xi_{1}\right\}$.

Now consider (4.7). Since $\underline{\phi}(\xi) \equiv 0$, using $(\mathrm{H} 2)$, and $\underline{\psi}(\xi) \geq 0$, we deduce that

$$
d_{1} \widetilde{\mathcal{N}}_{1}[\phi](\xi)-r_{1} \underline{\phi}(\xi)[1-\underline{\phi}(\xi)]+f(1-\underline{\phi}(\xi)) \underline{\psi}(\xi)-c \underline{\phi^{\prime}}(\xi)=f(1) \underline{\psi}(\xi) \geq 0 .
$$

Hence, for all $\xi \in \mathbb{R}$ (4.7) holds. It remains to check that 4.8 holds true. To do so, observe that for $\xi>\xi_{3}$, we have $\psi(\xi)=0$ and (4.8) holds true for $\xi>\xi_{3}$.

For $\xi<\xi_{3}$, one has $\underline{\psi}(\xi)=(-L \xi-q \sqrt{-\xi}) \mathrm{e}^{\lambda^{*} \xi}$. Furthermore, for all $z \in \mathbb{R}$, we have $\underline{\psi}(z) \geq(-L z-q \sqrt{-z}) \mathrm{e}^{\lambda^{*}} z$. Using (H3), 4.15), 4.16) and

$d_{2}\left[\int_{\mathbb{R}} \widetilde{J}_{2}\left(x_{1}\right) \mathrm{e}^{-\lambda^{*} x_{1}} \mathrm{~d} x_{1}-1\right]-c^{*} \lambda^{*}+r_{2}(\bar{\beta} f(1)-1)=0, \quad \int_{\mathbb{R}} \widetilde{J}_{2}\left(x_{1}\right) x_{1} \mathrm{e}^{-\lambda^{*} x_{1}} \mathrm{~d} x_{1}+c^{*}=0$,

it follows that

$$
\begin{aligned}
& d_{2} \widetilde{\mathcal{N}}_{2}[\underline{\psi}](\xi)-c^{*} \underline{\psi^{\prime}}(\xi)+r_{2} \underline{\psi}(\xi)[\bar{\beta} f(1-\bar{\phi}(\xi))-1-\underline{\psi}(\xi)] \\
\geq & d_{2}\left[\int_{\mathbb{R}} \widetilde{J}_{2}\left(x_{1}\right) \underline{\psi}\left(\xi-x_{1}\right) \mathrm{d} x_{1}-\underline{\psi}(\xi)\right]-c^{*} \underline{\psi}^{\prime}(\xi)+r_{2} \underline{\psi}(\xi)[\bar{\beta} f(1)(1-\bar{\phi}(\xi))-1-\underline{\psi}(\xi)] \\
\geq & d_{2} \int_{\mathbb{R}} \widetilde{J}_{2}\left(x_{1}\right)\left\{\left[-L\left(\xi-x_{1}\right)-q \sqrt{-\left(\xi-x_{1}\right)}\right] \mathrm{e}^{\lambda^{*}\left(\xi-x_{1}\right)}\right\} \mathrm{d} x_{1}-d_{2}(-L \xi-q \sqrt{-\xi}) \mathrm{e}^{\lambda^{*} \xi} \\
& +c^{*} L\left(1+\lambda^{*} \xi\right) \mathrm{e}^{\lambda^{*} \xi}+c^{*} q\left(\lambda^{*} \sqrt{-\xi}-\frac{1}{2 \sqrt{-\xi}}\right) \mathrm{e}^{\lambda^{*} \xi} \\
& +r_{2} \underline{\psi}(\xi)(\bar{\beta} f(1)-1)-r_{2} \bar{\beta} f(1) \bar{\psi}(\xi) \bar{\phi}(\xi)-r_{2} \bar{\psi}^{2}(\xi)
\end{aligned}
$$


Spreading Speed and Planar Waves for Predator-prey System

401

$$
\begin{aligned}
\geq & d_{2} \mathrm{e}^{\lambda^{*} \xi}\left[\int_{\mathbb{R}} \widetilde{J}_{2}\left(x_{1}\right)\left[-L\left(\xi-x_{1}\right)\right] \mathrm{e}^{-\lambda^{*} x_{1}} \mathrm{~d} x_{1}+L \xi\right]+c^{*} L\left(1+\lambda^{*} \xi\right) \mathrm{e}^{\lambda^{*} \xi} \\
& -q d_{2} \mathrm{e}^{\lambda^{*} \xi}\left[\int_{\mathbb{R}} \widetilde{J}_{2}\left(x_{1}\right) \sqrt{-\left(\xi-x_{1}\right)} \mathrm{e}^{-\lambda^{*} x_{1}} \mathrm{~d} x_{1}-\sqrt{-\xi}\right]+c^{*} q\left(\lambda^{*} \sqrt{-\xi}-\frac{1}{2 \sqrt{-\xi}}\right) \mathrm{e}^{\lambda^{*} \xi} \\
& +r_{2}(\bar{\beta} f(1)-1)\left[(-L \xi-q \sqrt{-\xi}) \mathrm{e}^{\lambda^{*} \xi}\right]-r_{2} \bar{\beta} f(1) \frac{K^{2}}{\bar{a}} \mathrm{e}^{\left(\lambda+\lambda_{*}\right) \xi}-r_{2} \frac{K^{2}}{\bar{a}^{2}} \mathrm{e}^{2 \lambda_{*} \xi} \\
= & -q d_{2} \mathrm{e}^{\lambda^{*} \xi}\left[\int_{\mathbb{R}} \widetilde{J}_{2}\left(x_{1}\right) \sqrt{-\left(\xi-x_{1}\right)} \mathrm{e}^{-\lambda^{*} x_{1}} \mathrm{~d} x_{1}-\sqrt{-\xi}\right]+c^{*} q\left(\lambda^{*} \sqrt{-\xi}-\frac{1}{2 \sqrt{-\xi}}\right) \mathrm{e}^{\lambda^{*} \xi} \\
& -r_{2}(\bar{\beta} f(1)-1) q \sqrt{-\xi} \mathrm{e}^{\lambda^{*} \xi}-r_{2} \bar{\beta} f(1) \frac{K^{2}}{\bar{a}} \mathrm{e}^{\left(\lambda+\lambda_{*}\right) \xi}-r_{2} \frac{K^{2}}{\bar{a}^{2}} \mathrm{e}^{2 \lambda_{*} \xi} \\
:= & \mathrm{e}^{\lambda^{*} \xi}\left[q I_{1}(\xi)-I_{2}(\xi)\right],
\end{aligned}
$$

where

$$
\begin{aligned}
I_{1}(\xi):= & -d_{2}\left[\int_{\mathbb{R}} \widetilde{J}_{2}\left(x_{1}\right) \sqrt{-\left(\xi-x_{1}\right)} \mathrm{e}^{-\lambda^{*} x_{1}} \mathrm{~d} x_{1}-\sqrt{-\xi}\right] \\
& +c^{*}\left(\lambda^{*} \sqrt{-\xi}-\frac{1}{2 \sqrt{-\xi}}\right)-r_{2}(\bar{\beta} f(1)-1) \sqrt{-\xi}, \\
I_{2}(\xi):= & r_{2} \bar{\beta} f(1) \frac{K^{2}}{\bar{a}} \mathrm{e}^{\left(\lambda+\lambda_{*}-\lambda^{*}\right) \xi}+r_{2} \frac{K^{2}}{\bar{a}^{2}} \mathrm{e}^{\left(2 \lambda_{*}-\lambda^{*}\right) \xi} .
\end{aligned}
$$

Next, to get the conclusion, we need to deduce a lower bound estimate of $I_{1}$ for $\xi<\xi_{3}$.

Using $\triangle\left(\lambda^{*}, c^{*}\right)=0$ and 4.18 , it follows that

$$
\begin{aligned}
I_{1}(\xi)= & -d_{2}\left\{\int_{\mathbb{R}} \widetilde{J}_{2}\left(x_{1}\right)\left[\sqrt{-\xi}+\sqrt{-\left(\xi-x_{1}\right)}-\sqrt{-\xi}\right] \mathrm{e}^{-\lambda^{*} x_{1}} \mathrm{~d} x_{1}-\sqrt{-\xi}\right\} \\
& +c^{*}\left(\lambda^{*} \sqrt{-\xi}-\frac{1}{2 \sqrt{-\xi}}\right)-r_{2}(\bar{\beta} f(1)-1) \sqrt{-\xi} \\
= & -d_{2}\left\{\int_{\mathbb{R}} \widetilde{J}_{2}\left(x_{1}\right)\left[\sqrt{-\left(\xi-x_{1}\right)}-\sqrt{-\xi}\right] \mathrm{e}^{-\lambda^{*} x_{1}} \mathrm{~d} x_{1}\right\}-\frac{c^{*}}{2 \sqrt{-\xi}} \\
= & d_{2}\left\{\int_{\mathbb{R}} \widetilde{J}_{2}\left(x_{1}\right)\left[\sqrt{-\xi}-\sqrt{-\left(\xi-x_{1}\right)}\right] \mathrm{e}^{-\lambda^{*} x_{1}} \mathrm{~d} x_{1}\right\}+\frac{d_{2}}{2 \sqrt{-\xi}} \int_{\mathbb{R}} \widetilde{J}_{2}\left(x_{1}\right) x_{1} \mathrm{e}^{-\lambda^{*} x_{1}} \mathrm{~d} x_{1} \\
:= & d_{2} \int_{\mathbb{R}} \widetilde{J}_{2}\left(x_{1}\right) Q\left(\xi, x_{1}\right) \mathrm{e}^{-\lambda^{*} x_{1}} \mathrm{~d} x_{1},
\end{aligned}
$$

where $Q\left(\xi, x_{1}\right)$ is defined by

$$
Q\left(\xi, x_{1}\right):=\frac{x_{1}}{2 \sqrt{-\xi}}+\sqrt{-\xi}-\sqrt{-\left(\xi-x_{1}\right)}
$$

Clearly

$$
\begin{aligned}
Q\left(\xi, x_{1}\right) & =\frac{x_{1}}{2 \sqrt{-\xi}}-\frac{x_{1}}{\sqrt{-\xi}-\sqrt{-\left(\xi-x_{1}\right)}}=\frac{x_{1}\left[\sqrt{-\left(\xi-x_{1}\right)}-\sqrt{-\xi}\right]}{2 \sqrt{-\xi}\left[\sqrt{-\left(\xi-x_{1}\right)}+\sqrt{-\xi}\right]} \\
& =\frac{x_{1}^{2}}{2 \sqrt{-\xi}\left[\sqrt{-\left(\xi-x_{1}\right)}+\sqrt{-\xi}\right]^{2}} .
\end{aligned}
$$


Because $J_{2}=0$ outside $\bar{B}_{G}(0)$ and

$$
2 \sqrt{-\xi}\left[\sqrt{-\left(\xi-x_{1}\right)}+\sqrt{-\xi}\right]^{2} \leq 8(-\xi+G)^{3 / 2} \text { for }\left|x_{1}\right|<G,
$$

we get the following lower estimate

$$
I_{1}(\xi) \geq \frac{d_{2}}{8(-\xi+G)^{3 / 2}} \int_{\mathbb{R}} \widetilde{J}_{2}\left(x_{1}\right) x_{1}^{2} \mathrm{e}^{-\lambda^{*} x_{1}} \mathrm{~d} x_{1} \quad \text { for } \xi<\xi_{3} .
$$

Finally, we use (4.17) and (4.19) to infer that

$$
d_{2} \tilde{\mathcal{N}}_{2}[\underline{\psi}](\xi)-c^{*} \underline{\psi}^{\prime}(\xi)+r_{2} \underline{\psi}(\xi)[\bar{\beta} f(1-\bar{\phi}(\xi))-1-\underline{\psi}(\xi)] \geq 0
$$

for all $\xi<\xi_{3}$. This completes the proof of the theorem.

The following theorem gives the proof of the existence of nonnegative solutions of system 4.4 with $c \geq c^{*}$ connecting the nontrivial state at $\xi=\infty$.

Theorem 4.6. Let $c \geq c^{*}$ and let $(\phi, \psi)$ be any solution provided by Theorems 4.4 or

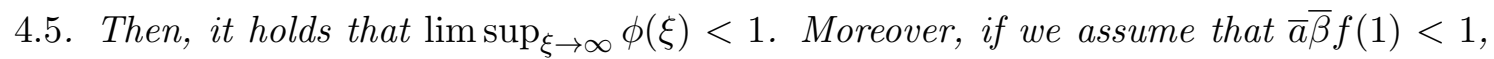
$\liminf _{\xi \rightarrow \infty} \psi(\xi)>0$ also hold true.

Proof. Let

$$
(U, V)(x, t):=(1-\phi, \psi)(x \cdot \nu+c t) .
$$

Since $V \leq \bar{\beta} f(1)-1$ in $\mathbb{R}^{N} \times \mathbb{R}$ and (H2), it follows from 1.1 that $U=U(x, t)$ satisfies

$$
\frac{\partial U(x, t)}{\partial t} \geq d_{1} \mathcal{N}_{1}[U(\cdot, t)](x)+r_{1} U(x, t)\{[1-\bar{a}(\bar{\beta} f(1)-1)]-U(x, t)\}, \quad x \in \mathbb{R}^{N}, t>0,
$$

such that $U(x, 0)=1-\phi(x \cdot \nu) \geq 0$ and $U \not \equiv 0$. Indeed, letting $x \cdot \nu \rightarrow-\infty$, we have $U(x, 0) \rightarrow 1$. Applying Proposition 2.1, we obtain that $U \geq z$, where $z$ is the solution to

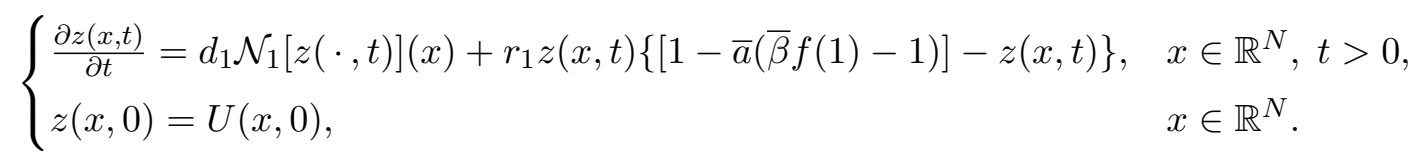

Also, from Proposition 2.3(ii), it follows that $z(x, t) \rightarrow 1-\bar{a}(\bar{\beta} f(1)-1)$ as $t \rightarrow \infty$ for all $x \in \mathbb{R}^{N}$. Hence, we obtain that

$$
\liminf _{t \rightarrow \infty} U(0, t) \geq \liminf _{t \rightarrow \infty} z(0, t)
$$

i.e.,

$$
\limsup _{t \rightarrow \infty} \phi(c t)=1-\liminf _{t \rightarrow \infty} U(0, t) \leq 1-\liminf _{t \rightarrow \infty} z(0, t)=\bar{a}(\bar{\beta} f(1)-1) .
$$

Since $c>0$, we conclude that

$$
\limsup _{\xi \rightarrow \infty} \phi(\xi) \leq \bar{a}(\bar{\beta} f(1)-1)<1
$$


To prove the second part, we first show a claim:

$$
D:=\sup _{\xi \in \mathbb{R}} \phi(\xi) \leq \bar{a}(\bar{\beta} f(1)-1)
$$

Indeed, by contradiction we assume that $D>\bar{a}(\bar{\beta} f(1)-1)$. Then, from 4.20 and $\phi(\xi) \rightarrow 0$ as $\xi \rightarrow-\infty$, there exists $\xi_{0} \in \mathbb{R}$ such that $\phi\left(\xi_{0}\right)=D$. Thus, $\phi\left(\xi_{0}\right)=D \geq \phi(\xi)$ for all $\xi \in \mathbb{R}$. Using $\phi(-\infty)=0, \phi \leq 1, \psi \leq \bar{\beta} f(1)-1$, and $f(x) \leq a x$, we obtain that

$$
\begin{aligned}
& d_{1} \widetilde{\mathcal{N}}_{1}[\phi]\left(\xi_{0}\right)-r_{1} \phi\left(\xi_{0}\right)\left[1-\phi\left(\xi_{0}\right)\right]+f\left(1-\phi\left(\xi_{0}\right)\right) \psi\left(\xi_{0}\right) \\
= & d_{1}\left[\int_{\mathbb{R}} \widetilde{J}_{1}\left(x_{1}\right) \phi\left(\xi_{0}-x_{1}\right) \mathrm{d} x_{1}-\phi\left(\xi_{0}\right)\right]-r_{1} \phi\left(\xi_{0}\right)\left(1-\phi\left(\xi_{0}\right)\right)+f\left(1-\phi\left(\xi_{0}\right)\right) \psi\left(\xi_{0}\right) \\
< & d_{1} \phi\left(\xi_{0}\right) \int_{\mathbb{R}} \widetilde{J}_{1}\left(x_{1}\right) \mathrm{d} x_{1}-d_{1} \phi\left(\xi_{0}\right)+r_{1}\left(1-\phi\left(\xi_{0}\right)\right)\left(\bar{a} \psi\left(\xi_{0}\right)-\phi\left(\xi_{0}\right)\right) \\
\leq & r_{1}\left(1-\phi\left(\xi_{0}\right)\right)[\bar{a}(\bar{\beta} f(1)-1)-D] \leq 0 .
\end{aligned}
$$

According to the first equation of (4.4), we conclude that $\phi^{\prime}\left(\xi_{0}\right)<0$ which contradicts $\phi^{\prime}\left(\xi_{0}\right)=0$. Hence, 4.21 holds.

From 4.21), (H3) and $\bar{a} \bar{\beta} f(1)<1$, it follows that the function $V(x, t)=\psi(x \cdot \nu+c t)$ satisfies

$$
\left\{\begin{aligned}
\frac{\partial V}{\partial t}(x, t) \geq & d_{2} \mathcal{N}_{2}[V(\cdot, t)](x) & & \\
& +r_{2} V(x, t)[(1-\bar{a} \bar{\beta} f(1))(\bar{\beta} f(1)-1)-V(x, t)], & & x \in \mathbb{R}^{N}, t>0 \\
V(x, 0)= & \psi(x), & & x \in \mathbb{R}^{N} .
\end{aligned}\right.
$$

Then, a similar argument above will deduce that

$$
\liminf _{t \rightarrow \infty} \psi(\xi) \geq(1-\bar{a} \bar{\beta} f(1))(\bar{\beta} f(1)-1)>0 .
$$

This completes the proof of the theorem.

The following theorem indicates that the nontrivial state at $\xi \rightarrow \infty$ is actually a coexisting state, if system (1.1) has a unique coexisting state and the wave tail converges at $\xi \rightarrow \infty$.

Theorem 4.7. Let $(U, V)$ be a traveling wave of (1.1) connecting $(1,0)$ and a nontrivial state. Assume that the limit

$$
(\bar{u}, \bar{v}):=\lim _{\xi \rightarrow \infty}(U, V)(\xi)
$$

exists. If the kinetic part of system (1.1) has a unique coexistence state $\left(U^{*}, V^{*}\right)$ satisfying

$$
\begin{aligned}
r_{1} U^{*}\left(1-U^{*}\right)-f\left(U^{*}\right) V^{*} & =0, \\
\beta f\left(U^{*}\right) V^{*}-r_{2} V^{*}\left(1+V^{*}\right) & =0,
\end{aligned}
$$

then $(\bar{u}, \bar{v})=\left(U^{*}, V^{*}\right)$. 
Proof. According to the assumption, we have $\left(U_{t}, V_{t}\right)(\xi) \rightarrow(0,0)$ as $\xi \rightarrow \infty$. By (4.1) and priori estimates, we obtain $0<\bar{u} \leq 1$ and $0<\bar{v} \leq \alpha$. To the aim, we only need to show that

$$
\left(\widetilde{J}_{1} * U\right)(\xi) \rightarrow \bar{u}, \quad\left(\widetilde{J}_{1} * V\right)(\xi) \rightarrow \bar{v} \quad \text { as } \xi \rightarrow \infty,
$$

if the kinetic part of system (1.1) has a unique coexistence state $\left(U^{*}, V^{*}\right)$.

Let $\epsilon \in(0, \bar{u})$. Because $U(+\infty)=\bar{u}$ and $\int_{\mathbb{R}} \widetilde{J}_{1}\left(x_{1}\right) \mathrm{d} x_{1}=1$, there exists $K_{0}>0$ large enough such that

$$
\int_{K_{0}}^{\infty} \widetilde{J}_{1}\left(x_{1}\right) \mathrm{d} x_{1}<\frac{\epsilon}{2} \quad \text { and } \quad \bar{u}-\frac{\epsilon}{2}<U(\xi)<\bar{u}+\frac{\epsilon}{2}, \quad \forall \xi \geq K_{0} .
$$

Denote

$$
\begin{aligned}
\int_{\mathbb{R}} \widetilde{J}_{1}\left(x_{1}\right) U\left(\xi-x_{1}\right) \mathrm{d} x_{1} & =\int_{-\infty}^{K_{0}} \widetilde{J}_{1}\left(x_{1}\right) U\left(\xi-x_{1}\right) \mathrm{d} x_{1}+\int_{K_{0}}^{\infty} \widetilde{J}_{1}\left(x_{1}\right) U\left(\xi-x_{1}\right) \mathrm{d} x_{1} \\
& :=I_{3}(\xi)+I_{4}(\xi)
\end{aligned}
$$

Using $\xi-x_{1} \geq K_{0}$ for $x_{1} \leq K_{0}$ and $\xi \geq 2 K_{0}, \int_{\mathbb{R}} \widetilde{J}_{1}\left(x_{1}\right) \mathrm{d} x_{1}=1$ and $U \leq 1$, it follows that

$$
I_{3}(\xi)+I_{4}(\xi) \leq\left(\bar{u}+\frac{\epsilon}{2}\right)+\frac{\epsilon}{2}=\bar{u}+\epsilon, \quad \forall \xi \geq 2 K_{0} .
$$

On the other hand, since $\bar{u} \leq 1$, we have

$$
I_{3}(\xi)+I_{4}(\xi) \geq I_{3}(\xi) \geq\left(\bar{u}-\frac{\epsilon}{2}\right)\left(1-\frac{\epsilon}{2}\right) \geq \bar{u}-\epsilon, \quad \forall \xi \geq 2 K_{0} .
$$

By 4.22 and 4.23 , we obtain

$$
\left(\widetilde{J}_{1} * U\right)(\xi) \rightarrow \bar{u}, \quad \xi \rightarrow \infty
$$

Using the same method, we get $\left(\widetilde{J}_{1} * V\right)(\xi) \rightarrow \bar{v}$ as $\xi \rightarrow \infty$. This completes the proof.

4.3. Nonexistence of planar wave solutions for $c<c^{*}$

Theorem 4.8. If $c \in\left(0, c^{*}\right)$, then (4.4) does not admit any nontrivial nonnegative solution $(\phi, \psi)$ satisfying 4.3 and $\lim _{\xi \rightarrow-\infty}(\phi(\xi), \psi(\xi))=(0,0)$.

Proof. To prove this result, by contradiction we assume that 4.4 admits a nontrivial nonnegative solution $(\phi, \psi)$ satisfying $\lim _{\xi \rightarrow-\infty}(\phi(\xi), \psi(\xi))=(0,0)$ and 4.3 for some wave speed $c_{1} \in\left(0, c^{*}\right)$. Here, we have $\phi>0$ and $\psi>0$ on $\mathbb{R}$.

Let $\epsilon \in(0,(\bar{\beta} f(1)-1) / 2)$ be given such that

$$
c_{2}:=\inf _{\lambda>0} \frac{d_{2}\left[\int_{\mathbb{R}} \widetilde{J}_{2}\left(x_{1}\right) \mathrm{e}^{\lambda x_{1}} \mathrm{~d} x_{1}-1\right]+r_{2}(\bar{\beta} f(1)-1-\epsilon)}{\lambda}>c_{1} .
$$


Because $\phi(-\infty)=0$, there exists a $\xi_{0} \in \mathbb{R}$ such that $\bar{\beta} f(1) \phi(\xi)<\epsilon$ for all $\xi \leq \xi_{0}$. Due to $\psi>0$ as $\xi>\xi_{0}$ and 4.3 , there exists $\delta>0$ such that $\inf _{\xi>\xi_{0}} \psi(\xi) \geq \delta$.

Define $M_{2}>1$ by $\delta\left(M_{2}-1\right)=\bar{\beta} f(1)$. Then, since $\phi \leq 1$, we get

$$
\bar{\beta} f(1) \phi(\xi) \leq\left(M_{2}-1\right) \psi(\xi) \text { for all } \xi \geq \xi_{0}
$$

Using (H3), we deduce that

$$
c_{1} \psi^{\prime}(\xi) \geq d_{2} \widetilde{\mathcal{N}}_{2}[\psi](\xi)+r_{2} \psi(\xi)\left[\bar{\beta} f(1)-1-\epsilon-M_{2} \psi(\xi)\right], \quad \xi \in \mathbb{R},
$$

and the function $V(x, t):=\psi\left(x \cdot \nu+c_{1} t\right)$ satisfies

$$
\begin{cases}\frac{\partial V}{\partial t}(x, t) \geq d_{2} \mathcal{N}_{2}[V(\cdot, t)](x)+r_{2} V(x, t)\left[\bar{\beta} f(1)-1-\epsilon-M_{2} V(x, t)\right], & x \in \mathbb{R}^{N}, t>0 \\ V(x, 0)=\psi(x)>0, & x \in \mathbb{R}^{N} .\end{cases}
$$

Denote $c:=\left(c_{1}+c_{2}\right) / 2<c_{2}$. Applying Propositions 2.1 and 2.3(ii), we can infer that

$$
\liminf _{t \rightarrow \infty} V_{\|x\|<c t}(x, t) \geq \frac{\bar{\beta} f(1)-1-2 \epsilon}{M_{2}}>0 .
$$

Letting $x_{1}=-\frac{c_{1}+c_{2}}{2} t e_{1}$, where $e_{1}=(1,0, \ldots, 0)$, we have $\left\|x_{1}\right\|<c t$. Clearly,

$$
-\left(c_{2}+c_{1}\right) t / 2+c_{1} t=\left(c_{1}-c_{2}\right) t / 2 \rightarrow-\infty \quad \text { as } t \rightarrow \infty .
$$

Thus, it follows from 4.24 that

$$
\liminf _{\xi \rightarrow-\infty} \psi(\xi)=\liminf _{t \rightarrow \infty} V\left(-\frac{c_{1}+c_{2}}{2} t, t\right) \geq \frac{\bar{\beta} f(1)-1-2 \epsilon}{M_{2}}>0,
$$

which contradicts the limit condition $\psi(-\infty)=0$. This completes the proof.

\subsection{Minimal wave speed}

In this subsection, we conclude that the spreading speed of the predatory is the minimal wave speed $c^{*}$.

Theorem 4.9. In addition to (1.3), assume that a $\beta f(1)<r_{1} r_{2}$ and $d_{2}<\beta f(1)-r_{2}$, and that $J_{2}$ has a compact support. Then, (1.1) admits planar wave solution connecting $(1,0)$ to a nontrivial state with speed $c \in \mathbb{R}$ if and only if $c \geq c^{*}$.

Proof. According to the results in Sections 4.2 and 4.3 , in order to prove the theorem, we only need to prove that, under the parameter condition $d_{2}<\beta f(1)-r_{2}$, the nonnegative planar wave solution $(\phi, \psi)$ of system 4.4 combined with $(\phi, \psi)(-\infty)=(0,0)$ and 4.3 . satisfies $c>0$. Indeed, by contradiction, we assume that (4.4) admits a nonnegative solution $(\phi, \psi)$ with speed $c \leq 0$ satisfying $(\phi, \psi)(-\infty)=(0,0)$ and 4.3 . 
Due to $(\phi, \psi)(-\infty)=(0,0)$ and $(\mathrm{H} 3)$, there exists a sufficiently large constant $M_{3}$ such that for all $\xi<-M_{3}$,

$$
\begin{aligned}
r_{2}[\bar{\beta} f(1-\phi(\xi))-1-\psi(\xi)] & \geq r_{2}[\bar{\beta} f(1)-\bar{\beta} f(1) \phi(\xi)-1-\psi(\xi)] \\
& >L_{1}:=\frac{r_{2}(\bar{\beta} f(1)-1)}{2}+\frac{d_{2}}{2} .
\end{aligned}
$$

Since $\widetilde{J}_{2} \geq 0$ and $\psi>0$, it follows from 4.4 and 4.25 that for all $\xi<-M_{3}$,

$$
\begin{aligned}
c \psi^{\prime}(\xi) & =d_{2} \int_{\mathbb{R}} \widetilde{J}_{2}\left(\xi-x_{1}\right) \psi\left(x_{1}\right) \mathrm{d} x_{1}-d_{2} \psi(\xi)+r_{2} \psi(\xi)[\bar{\beta} f(1-\phi(\xi))-1-\psi(\xi)] \\
& \geq r_{2} \psi(\xi)[\bar{\beta} f(1)-\bar{\beta} f(1) \phi(\xi)-1-\psi(\xi)]-d_{2} \psi(\xi) \\
& \geq L_{1} \psi(\xi)-d_{2} \psi(\xi) \\
& =\left(L_{1}-d_{2}\right) \psi(\xi) .
\end{aligned}
$$

Moreover, according to the condition $d_{2}<\beta f(1)-r_{2}=r_{2}(\bar{\beta} f(1)-1)$, we have $L_{2}:=L_{1}-d_{2}>0$. Integrating from $-\infty$ to $y \leq-M_{3}$ for the above inequality, we have

$$
c \psi(y) \geq L_{2} \int_{-\infty}^{y} \psi(\xi) \mathrm{d} \xi, \quad \forall y \leq-M_{3} .
$$

Thus, we have $c>0$ which contradicts $c \leq 0$. This completes the proof.

\section{Numerical simulations}

In this section, we illustrate the main results by using numerical simulations.

For simplicity, we consider $N=1, f(U)=\frac{U}{1+U}$, that is, the system (1.1) becomes

$$
\left\{\begin{array}{rl}
\frac{\partial U}{\partial t}(x, t)= & d_{1} \mathcal{N}_{1}[U(\cdot, t)](x)+r_{1} U(x, t)(1-U(x, t)) \\
& -\frac{U(x, t)}{1+U(x, t)} V(x, t), \\
\frac{\partial V}{\partial t}(x, t)= & d_{2} \mathcal{N}_{2}[V(\cdot, t)](x)+\beta \frac{U(x, t)}{1+U(x, t)} V(x, t) \\
& -r_{2} V(x, t)(1+V(x, t)),
\end{array} \quad x \in \mathbb{R}, t>0 .\right.
$$

We take the kernel function $J_{i}(x)=J_{\varrho}(x)=\frac{1}{\sqrt{2 \pi \varrho^{2}}} \mathrm{e}^{\frac{-x^{2}}{2 \varrho^{2}}}(i=1,2)$. In addition, the parameters of system (5.1) are taken as: $d_{1}=d_{2}=1, r_{1}=4, r_{2}=1, \varrho=1, \beta=5$. It is easy to know that the system (5.1) has three constant equilibrium states: $E_{0}=(0,0)$, $E_{1}=(1,0)$ and $E^{*}=\left(U^{*}, V^{*}\right)=(0.8271,1.2634)$.

First, we use numerical simulations to illustrate the spreading speed of the predator. We choose $\Omega_{1}=[-150,150]$ and initial conditions

$$
U(x, 0)=1, \quad v_{0}(x)= \begin{cases}\frac{1}{5}, & x=0, \\ \frac{1}{5}\left(\frac{1}{5}-|x|\right), & 0<|x| \leq 5, \\ 0, & |x|>5 .\end{cases}
$$


With the help of MATLAB, we obtain the numerical solution $(U, V)$ of system (5.1) under the initial conditions 5.2 (see Figure 5.1). Figure 5.1 shows that there are a small number of predators $V$ near the origin. These predators spread in space over time and feed on the aborigine prey $U$ (the density of $U$ is 1 ), after a period of time, the density of prey decreases and the density of the predator increases. If let $x_{ \pm}^{a}(t)$ denote the spatial position when the species $V$ diffuses to the density $a$ (when spreading to the left to the density $a$, it is represented by $x_{-}^{a}(t)$, and to the right is represented by $\left.x_{+}^{a}(t)\right)$, as shown in Figure 5.2. The spreading speed can be defined as $c^{*}= \pm \lim _{t \rightarrow \infty} \frac{d x_{ \pm}^{a}(t)}{d t}, \forall a \in(0,1)$. From Figures 5.2 and 5.3 , we get the spreading speed of the predator is about $c^{*}=2.118$.
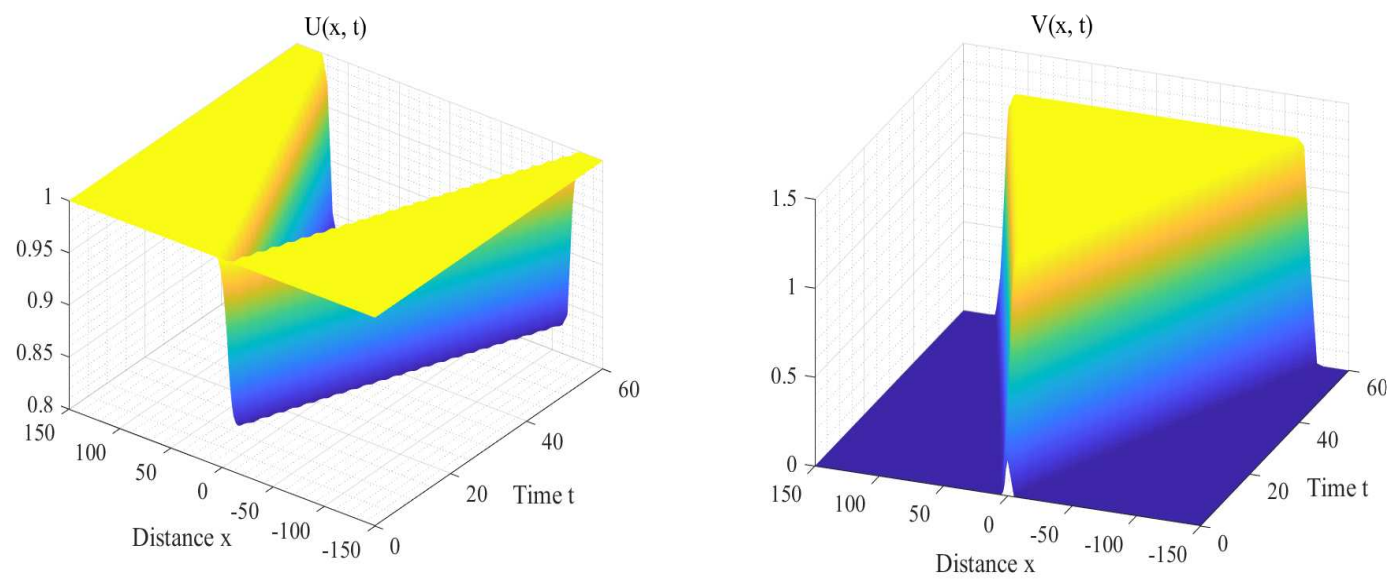

Figure 5.1: Numerical solution of system (5.1) under initial conditions 5.2 .
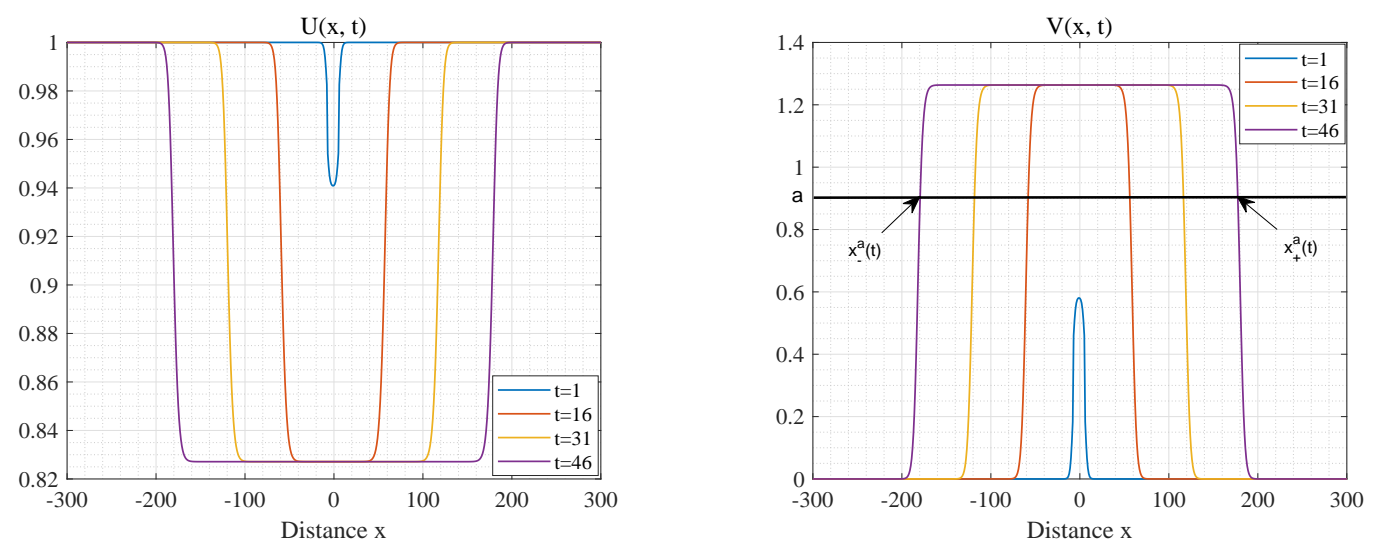

Figure 5.2: Figures of the solution in Figure 5.1 at time $t=1,16,31,46$.

Now, we use numerical simulations to study the planar wave solutions of system (5.1). 
We choose $\Omega_{2}=[-400,400]$ and the initial datum

$$
\left\{\begin{array}{l}
U(x, 0)=\left(1-U^{*}\right)\left(1-\frac{1}{4}(1+\tanh (x))^{2}\right)+U^{*}, \\
v_{0}(x)=V^{*}\left(1-\frac{1}{4}(1-\tanh (x))^{2}\right),
\end{array} \quad x \in[-400,400] .\right.
$$
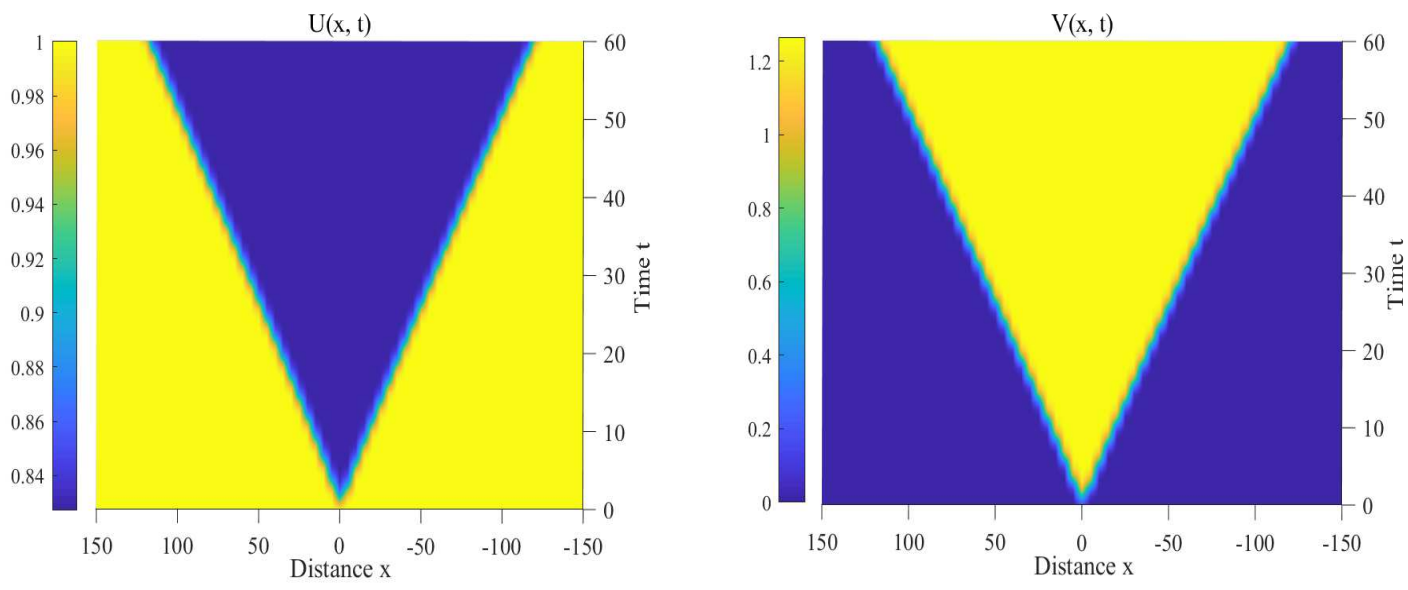

Figure 5.3: The projections of $U(x, t)$ and $V(x, t)$ on the $x o t$ plane in Figure 5.1

From Theorems 4.4, 4.5 and 4.6, system (5.1) admits a planar wave connecting the predator-free state $(1,0)$ and a nontrivial state with speed $c \geq c^{*}=2.118$. According to Theorem 4.9, we know that $c^{*}$ is also the minimal wave speed of the invasion planar waves. Through numerical simulations (see Figure 5.4), we can get the same result as Theorem 4.7, namely, the solution of system (5.1) eventually converge to the positive equilibrium $\left(U^{*}, V^{*}\right)$, which implies that the species $U$ and $V$ will coexist.
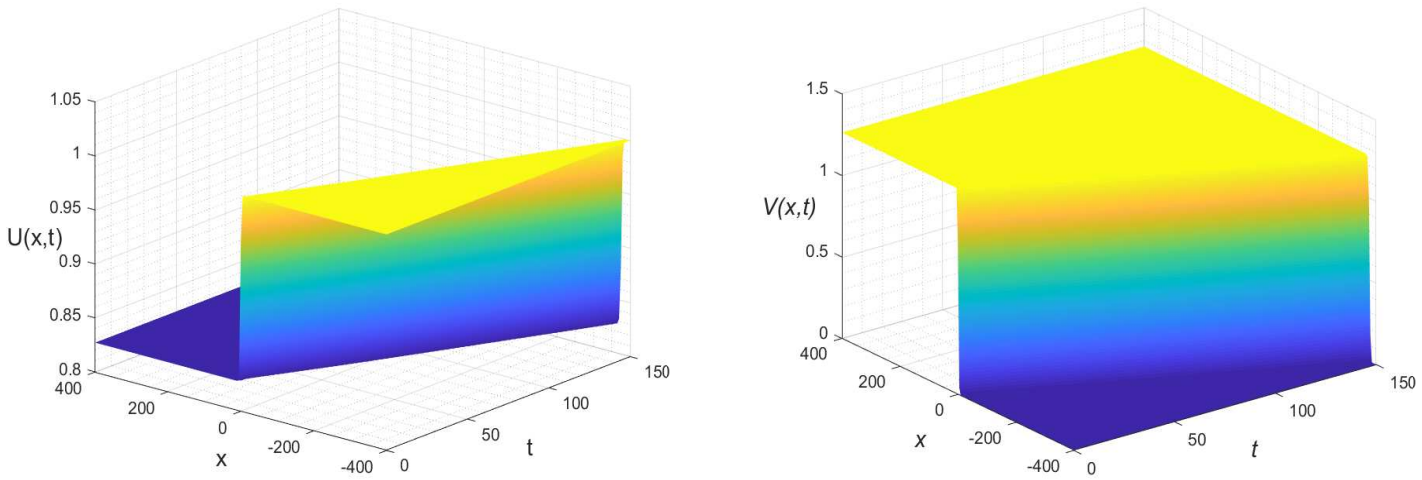

Figure 5.4: The solutions of system (5.1) with initial (5.3).

Here, we give some explanations of the ecological phenomena related to these results. In the actual biological environment, at the beginning, there was only the aborigine species 
$U$ with a density at its carrying capacity. After some time, the exotic species $V$ invades the system (5.1) by feeding on species $U$. Then based on our results, the two species will eventually coexist.

\section{Acknowledgments}

We are very grateful to the anonymous referee for careful reading and helpful suggestions which led to an improvement of our original manuscript. This work is supported by the National Natural Science Foundation of China (Nos. 11771044, 11871007, 12171039 and 12001502).

\section{References}

[1] D. G. Aronson and H. F. Weinberger, Multidimensional nonlinear diffusion arising in population genetics, Adv. in Math. 30 (1978), no. 1, 33-76.

[2] Y.-Y. Chen, J.-S. Guo and C.-H. Yao, Traveling wave solutions for a continuous and discrete diffusive predator-prey model, J. Math. Anal. Appl. 445 (2017), no. 1, $212-239$.

[3] H. Cheng and R. Yuan, Existence and stability of traveling waves for Leslie-Gower predator-prey system with nonlocal diffusion, Discrete Contin. Dyn. Syst. 37 (2017), no. $10,5433-5454$.

[4] O. Diekmann, Run for your life: A note on the asymptotic speed of propagation of an epidemic, J. Differential Equations 33 (1979), no. 1, 58-73.

[5] F.-D. Dong, W.-T. Li and G.-B. Zhang, Invasion traveling wave solutions of a predator-prey model with nonlocal dispersal, Commun. Nonlinear Sci. Numer. Simul. 79 (2019), 104926, 17 pp.

[6] A. Ducrot, J.-S. Guo, G. Lin and S. Pan, The spreading speed and the minimal wave speed of a predator-prey system with nonlocal dispersal, Z. Angew. Math. Phys. 70 (2019), no. 5, Paper No. 146, 25 pp.

[7] J. Fang and X.-Q. Zhao, Traveling waves for monotone semiflows with weak compactness, SIAM J. Math. Anal. 46 (2014), no. 6, 3678-3704.

[8] W. Huang, Traveling wave solutions for a class of predator-prey systems, J. Dynam. Differential Equations 24 (2012), no. 3, 633-644.

[9] L. I. Ignat and J. D. Rossi, A nonlocal convection-diffusion equation, J. Funct. Anal. 251 (2007), no. 2, 399-437. 
[10] Y. Jin and X.-Q. Zhao, Spatial dynamics of a periodic population model with dispersal, Nonlinearity 22 (2009), no. 5, 1167-1189.

[11] X.-S. Li, S. Pan and H.-B. Shi, Minimal wave speed in a dispersal predator-prey system with delays, Bound. Value Probl. 2018, Paper No. 49, 26 pp.

[12] X. Liang and X.-Q. Zhao, Asymptotic speeds of spread and traveling waves for monotone semiflows with applications, Comm. Pure Appl. Math. 60 (2007), no. 1, 1-40.

[13] Z. Ma, Stability of traveling wave solutions and spreading speed for several reactiondiffusion equations, $\mathrm{PhD}$ thesis, Beijing Normal University, 2019.

[14] Z. Ma, X. Wu, R. Yuan and Y. Wang, Multidimensional stability of planar waves for delayed reaction-diffusion equation with nonlocal diffusion, J. Appl. Anal. Comput. 9 (2019), no. 3, 962-980.

[15] Z. Ma, R. Yuan, Y. Wang and X. Wu, Multidimensional stability of planar traveling waves for the delayed nonlocal dispersal competitive Lotka-Volterra system, Commun. Pure Appl. Anal. 18 (2019), no. 4, 2069-2091.

[16] S. Pan, Traveling wave solutions in nonlocal dispersal models with nonlocal delays, J. Korean Math. Soc. 51 (2014), no. 4, 703-719.

[17] H. F. Weinberger, Long-time behavior of a class of biological models, SIAM J. Math. Anal. 13 (1982), no. 3, 353-396.

[18] H. F. Weinberger, M. A. Lewis and B. Li, Analysis of linear determinacy for spread in cooperative models, J. Math. Biol. 45 (2002), no. 3, 183-218.

Min Zhao

Laboratory of Mathematics and Complex Systems (Ministry of Education), School of Mathematical Sciences, Beijing Normal University, Beijing 100875, China

E-mail address: minzhao@mail.bnu.edu.cn

Zhaohai Ma

School of Science, China University of Geosciences, Beijing 100083, China

E-mail address: zhaohaima@cugb.edu.cn

Rong Yuan

Laboratory of Mathematics and Complex Systems (Ministry of Education), School of Mathematical Sciences, Beijing Normal University, Beijing 100875, China

E-mail address: ryuan@bnu.edu.cn 